\title{
Excited State Vibrations of Isotopically Labelled FMN Free and Bound to a LOV Protein
}

James N. Iuliano, ${ }^{1 \ddagger}$ Christopher R. Hall, ${ }^{2 \ddagger}$ Dale Green, ${ }^{2 \ddagger}$ Garth A. Jones, ${ }^{2}$ Andras Lukacs, ${ }^{3}$ Boris Illarionov, ${ }^{4}$ Adelbert Bacher, ${ }^{4,5}$ Markus Fischer, ${ }^{4}$ Jarrod B. French, ${ }^{1}$ Peter J. Tonge ${ }^{1 *}$ and Stephen R. Meech $^{2 *}$

${ }^{1}$ Department of Chemistry, Stony Brook University, Stony Brook, New York 11794-3400, United States, ${ }^{2}$ School of Chemistry, University of East Anglia, Norwich NR4 7TJ, U.K., ${ }^{3}$ Department of Biophysics, Medical School, University of Pecs, Szigeti ut 12, 7624 Pecs, Hungary, ${ }^{4}$ Institut für Biochemie und Lebensmittelchemie, Universität Hamburg, Grindelallee 117, D-20146 Hamburg, Germany ${ }^{5}$ Department of Chemistry, Technical University of Munich, 85747 Garching, Germany

\section{Abstract}

Flavoproteins are important blue light sensors in photobiology and play a key role in optogenetics. The characterization of their excited state structure and dynamics is thus an important objective. Here we present a detailed study of excited state vibrational spectra of flavin mononucleotide (FMN), in solution and bound to the LOV-2 (Light-Oxygen-Voltage) domain of Avena sativa phototropin. Vibrational frequencies are determined for the optically excited singlet state and the reactive triplet state, through resonant ultrafast femtosecond stimulated Raman spectroscopy (FSRS). To assign the observed spectra, vibrational frequencies of the excited states are calculated using density functional theory, and both measurement and theory are applied to four different isotopologues of FMN. Excited state mode assignments are refined in both states and their sensitivity to deuteration and protein environment are investigated. We show that resonant FSRS provides a useful tool for characterizing photoactive flavoproteins, and is able to highlight chromophore localized modes, and to record hydrogen/deuterium exchange.

$\ddagger J N I, C R H$ and DG contributed equally to this work through protein spectroscopy, FSRS development and calculations respectively. 
*Address for correspondence: peter.tonge@stonybrook.edu; s.meech@uea.ac.uk 


\section{Introduction}

The isoalloxazine moiety of flavin mononucleotide (FMN), a tricyclic heterocycle, is the chromophore responsible for the yellow pigmentation of flavoproteins. It is the common core of the different flavin cofactors (e.g. riboflavin, FMN, FAD) usually non-covalently bound in flavoproteins. ${ }^{1}$ In many proteins the flavin is a redox active element involved in electron transfer reactions. ${ }^{1}$ However, in a number of flavoproteins the flavin serves as a photoactive element, involved in mediating a variety of light driven processes, including DNA repair (photolyases), phototaxis (BLUF domains) and phototropism (LOV domains). ${ }^{2-5}$ This has driven much of the recent interest in the photochemistry and photophysics of flavins and flavoproteins, which accelerated with the discovery that photoactive flavoproteins may be used in optogenetics, where their ability to modify gene expression in a light sensitive fashion has been recruited to optically control cellular activity. ${ }^{6}$

The investigation of flavoprotein photophysics necessarily entails the study of the electronically excited states of isoalloxazine. Transient absorption has been used to probe the excited state dynamics of a number of flavin cofactors and flavoproteins, yielding a detailed picture of the evolution of excited state populations and thus the rates of product formation on the femtosecond to nanosecond time scale..$^{7-8}$ Structural information on excited state dynamics has been provided by transient infra-red (TRIR) measurements from ultrafast to seconds timescales. ${ }^{9-13}$ Significantly, TRIR experiments have the ability to probe the response to optical excitation of both the flavin moiety and the surrounding protein residues, thus providing a more complete picture of protein function. ${ }^{14}$ One challenge in TRIR experiments is separating the contributions of the chromophore from those of the surrounding amino acid residues. Understanding both is vital to unravelling the protein's signalling mechanism. In TRIR this separation has been addressed through the study of isotopically substituted flavins, by isotope editing key protein residues or by site specific introduction of IR marker modes, using noncanonical amino acid substitution. ${ }^{13,15-17}$ 
More recently the technique of femtosecond stimulated Raman spectroscopy (FSRS) has been developed to measure the vibrational Raman spectrum of excited electronic states and photoproducts. ${ }^{18-19}$ In addition to its ability to record transient real-time Raman spectra, FSRS can exploit resonance enhancements to probe specifically chromophore excited states. ${ }^{20}$ In photobiology resonant FSRS offers the opportunity of selectively recording spectra in specific electronic states (e.g. singlet and triplet, see below) and of separately measuring chromophore and protein modes. As such, FSRS (and related Raman experiments) have the potential to become a powerful tool in time resolved photobiology, which will be complementary to TRIR. In particular (i) studies in $\mathrm{H}_{2} \mathrm{O}$ buffer are possible using Raman methods, while for TRIR in $\mathrm{H}_{2} \mathrm{O}$ absorption near the amide region can seriously degrade signal-to-noise, leading to $\mathrm{D}_{2} \mathrm{O}$ being the favoured solvent (ii) very large protein complexes and whole cells have strong IR absorption, which can degrade signal-to-noise by a similar mechanism to $\mathrm{H}_{2} \mathrm{O}$ and (iii) Raman experiments yield a wide spectral range in a single experiment, while TRIR is typically restricted to a few hundred wavenumbers.

In an important paper Ernsting and co-workers demonstrated that FSRS yields the Raman spectrum of the excited singlet state of riboflavin and FAD with good signal-to-noise. ${ }^{21}$ They investigated mode assignments in the $S_{1}$ state through TD-DFT calculations, including four water molecules to represent a hydrogen-bonding environment, as well as using a polarizable continuum model (PCM) for solvent effects. We extended the FSRS measurements to photoactive flavoproteins, specifically the blue light using flavin (BLUF) domain protein AppA, where the sensitivity of the FSRS signal to the dark or light adapted (signalling) state of the protein was investigated. ${ }^{22}$ Recently Andrikopoulos et al. reported the FSRS spectrum of FMN in both its singlet and triplet states, and again endeavoured to assign the observed modes through DFT calculations. ${ }^{23}$ In this work we present a detailed assignment of the FSRS spectrum of FMN in its singlet and triplet states through the study of four different isotopologues of isoalloxazine, complemented by the corresponding TD-DFT calculations. Isotope shifts aid assignment of the observed bands to calculated modes, which are generally more numerous. Further we extend this approach to an investigation of the spectra of the recombinant 
LOV-2 (Light-Oxygen-Voltage) domain of Avena sativa phototropin (subsequently designated AsLOV2) that has been studied earlier by transient IR. ${ }^{24-25}$ The primary event in LOV domain photochemistry is intersystem crossing to the triplet state, which then undergoes a reaction with an adjacent cysteine residue. ${ }^{26}$ The subsequent change in protein structure, an unbinding and uncoiling of an $\alpha$-helix, ${ }^{27}$ initiates signalling. The LOV-2 domain is involved in controlling phototropism, and has also been adopted as an optogenetic element..$^{28-30}$

\section{Methods}

(i) Femtosecond Stimulated Raman. FSRS spectra were measured using an instrument described in detail elsewhere..$^{31-32}$ The $800 \mathrm{~nm}$ output of a $1 \mathrm{kHz}$ Ti:Sapphire laser was divided to pump two optical parametric amplifiers (OPA) and as input to a second harmonic bandwidth compressor (SHBC). The first OPA generated $80 \mathrm{fs}$ 'actinic' pump pulses at $450 \mathrm{~nm}(1 \mu \mathrm{J}, 170 \mu \mathrm{m}$ spot size) to photochemically excite the sample. The second OPA generates $100 \mathrm{fs}$ pulses at $1100 \mathrm{~nm}$ which are then focused onto a $2 \mathrm{~mm} \mathrm{CaF}_{2}$ window to generate a white light continuum (480-1000 nm, $30 \mu \mathrm{m}$ spot size) which act as the FSRS 'Raman probe'. The picosecond $400 \mathrm{~nm}$ output of the SHBC is used to pump a third OPA which generates narrowband (ca $\left.10 \mathrm{~cm}^{-1}\right)$ picosecond 'Raman Pump' pulses. The pulse is tunable throughout the vis and near IR. In the present experiment it was centered at $750 \mathrm{~nm}$ (4 $\mu \mathrm{J} 100 \mu \mathrm{m}$ spot size), a wavelength which was selected to be resonant with the excited state transient absorption of both singlet and triplet states of FMN; as described by Andrikopoulos et al there is a broad transient absorption at ca $800 \mathrm{~nm}$ for $\mathrm{S}_{1}$, which evolves into a more triplet-triplet absorption with a much larger transition dipole moment and a peak at $712 \mathrm{~nm} \cdot{ }^{23}$ Pulses were overlapped and focused to the sample position and the stimulated Raman signal was collected in the phase matched directions and dispersed in a SPEX 500M spectrometer with CCD detector. Optical choppers were used to modulate the actinic and Raman pump pulses resulting in four sets of pulse 
sequences (a) Actinic Pump-Probe+Raman, (b) Raman+Probe, (c) Actinic Pump+Probe, and (d) probe only such that the excited state FSRS signals can be extracted from the transient absorption using:

Raman Gain $=\log \left(\frac{\text { Pump }+ \text { Probe }+ \text { Raman }}{\text { Probe }+ \text { Raman }}\right)$,

as described elsewhere. ${ }^{31}$ Spectra were calibrated using neat cyclohexane. The bandwidth was measured as $<20 \mathrm{~cm}^{-1}$. In tables presented below we report the wavenumber maxima of the observed bands, and estimate a $3 \mathrm{~cm}^{-1}$ shift as detectable. Samples (optical density 0.5 at $450 \mathrm{~nm}$ ) were flowed through a $200 \mu \mathrm{m}$ path length $\mathrm{CaF}_{2}$ cell at a rate of $\sim 2 \mathrm{~mL} / \mathrm{min}$. All measurements were performed in $20 \mathrm{mM}$ Tris pH8.0, $150 \mathrm{mM} \mathrm{NaCl}$ unless otherwise indicated.

(ii) TD-DFT Calculations. The isoalloxazine chromophore in FMN was modelled in the form of lumiflavin, simplifying the ribityl-5'-phosphate in FMN to a methyl group. The ground state, $\mathrm{S}_{0}$, was optimized using DFT at the B3LYP $33-34 / T Z V P^{35}$ level of theory and the optimized structure was characterised using harmonic frequency analysis at $298.15 \mathrm{~K}$ and $1 \mathrm{~atm}$. In the style of Ernsting et al. ${ }^{21}$ the chromophore is solvated by four explicit water molecules positioned around the polar end of the isoalloxazine moiety in addition to including a polarizable continuum model (PCM) $)^{36-37}$ for water. The excited states $S_{1}$ and $T_{1}$ were optimized at the same level of theory, using TD-DFT for $S_{1}$ and unrestricted DFT for $T_{1}$. These stationary points were also characterized by harmonic analysis and found to correspond to minima. The optimized structure for the ground state, $\mathrm{S}_{0}$, is shown in Figure 1. The microsolvation layer provided by the four water molecules represents the $\mathrm{H}$-bonding interaction, typical of the protein environment. In agreement with experiment, greater stabilisation of non-bonding and unoccupied $\pi^{*}$ orbitals localised the polar end of isoalloxazine results in a red-shift of $\pi \pi^{*}$ versus a blue-shift of $n \pi^{*}$ transitions, such that the lowest energy transition for the explicitly solvated chromophore is an allowed $\pi \pi^{*}$ and any electronic coupling between these states is reduced. ${ }^{21,38-39} \mathrm{IR}$ and Raman spectra were calculated for five isotopologues ([U- $\left.{ }^{15} \mathrm{~N}_{4}\right]-\mathrm{FMN}$; $[4,10 \mathrm{a}-$ $\left.{ }^{13} C_{2}\right]$-FMN; $\left[2,4 a-{ }^{13} C_{2}\right]-F M N ;\left[2-{ }^{13} C_{1}\right]-F M N$ and $\left.\left[4 a-{ }^{13} C_{1}\right]-F M N\right)$ at the optimized geometries of the three electronic states considered $\left(S_{0}, S_{1}\right.$ and $\left.T_{1}\right)$. Vibrational analysis was also repeated for the 
excited states $\left(\mathrm{S}_{1}\right.$ and $\left.\mathrm{T}_{1}\right)$ of FMN on deuteration of the only exchangeable hydrogen of the isoalloxazine, $\mathrm{N} 3 \mathrm{H}$ (see also Figure 2a), alone and on deuteration of both $\mathrm{N} 3 \mathrm{H}$ and the four explicit water molecules. All calculations were completed using Gaussian $16 .{ }^{40}$ The wavenumbers reported are unscaled, as it is not yet clearly established whether the 0.97 factor required for the ground state at this level of theory is also applicable to the excited state(s).

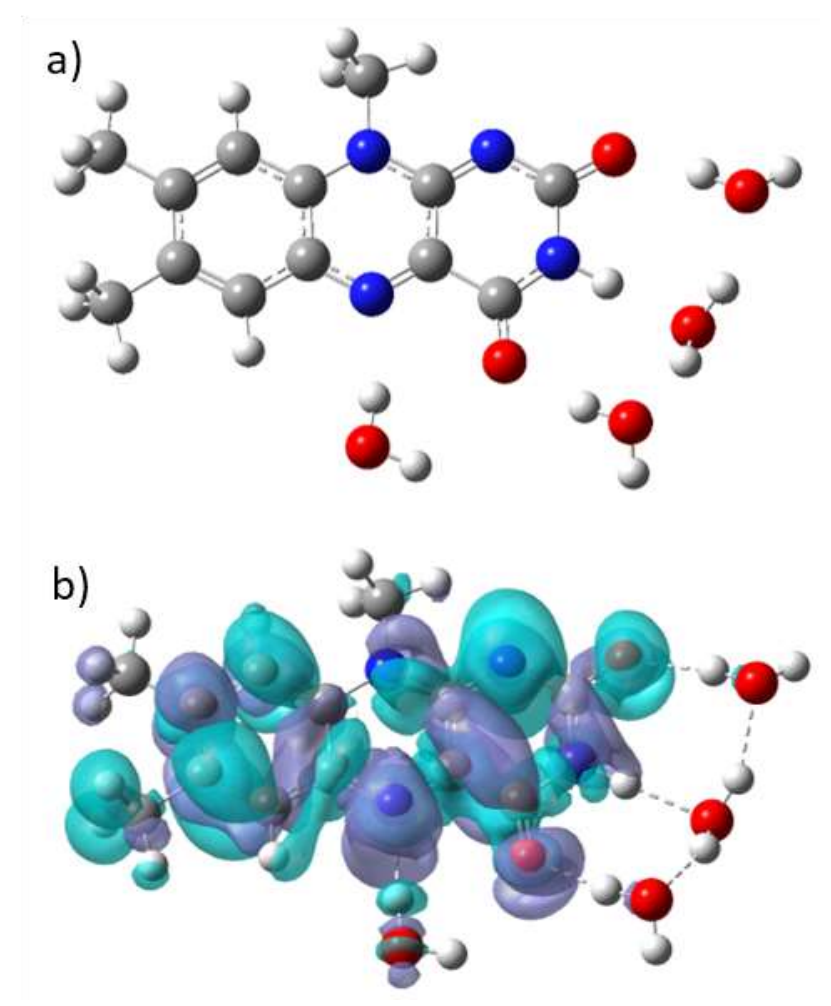

Figure 1: (a) Ground state ( $\left.S_{0}\right)$ geometry of lumiflavin solvated by four water molecules as well as the PCM, optimized at the B3LYP/TZVP level of theory. (b) Electron density difference map for $\mathrm{S}_{1}$-So, where dark (light) blue indicates regions of increased (decreased) electron density.

Although the focus of this paper is on the vibrational spectra of the excited states, we present in Figure $1 b$ the electron density difference map between $S_{1}$ and $S_{0}$, as an aid to understanding wavenumber shifts between states. This illustrates the potentially important role of $\mathrm{H}$-bonding interactions, most notably at N5 where the calculated distance to the water oxygen contracts by $9.3 \mathrm{pm}$ in $\mathrm{S}_{1}$. As described below, the $\mathrm{H}$-bond environment modifies the vibrational spectra of the electronically excited isoalloxazine moiety (and vice versa). ${ }^{14}$ 
(iii) Reagents. ${ }^{13} \mathrm{C}$-labeled riboflavin isotopologs were synthesized using the method reported by Tishler et al. ${ }^{41}$ Isotope enrichments were approximately $99 \%$. [U- $\left.{ }^{15} \mathrm{~N}_{4}\right]$ riboflavin was obtained by fermentation using a recombinant $E$. coli strain that was grown with ${ }^{15} \mathrm{NH}_{4} \mathrm{Cl}$ as the single nitrogen source..$^{42}$ Enzyme-catalyzed phosphorylation of riboflavin isotopologs was performed as described elsewhere $e^{43}$

(iv) Protein Preparation. A synthetic open reading frame specifying an $\mathrm{N}$-terminal polyhistidine tag ${ }^{44}$ recombinant Escherichia coli strain (UniProtKB O49003). The recombinant AsLOV2 protein was purified as described previously. ${ }^{24}$

(v) Ligand exchange. Recombinant AsLOV2 $(20 \mathrm{mg}$ in $8 \mathrm{ml}$ of $50 \mathrm{mM}$ Tris hydrochloride, $\mathrm{pH}$ 8.0, containing $200 \mathrm{mM} \mathrm{NaCl}, 50 \mathrm{mM}$ imidazole and $0.2 \% \mathrm{NaN}_{3}$ was applied to a column of chelating Sepharose $\left(\mathrm{Ni}^{2+}\right.$ form, $\left.1 \mathrm{~cm} \times 6 \mathrm{~cm}\right)$ that had been equilibrated with $50 \mathrm{mM}$ Tris hydrochloride, $\mathrm{pH}$ 8.0, containing $400 \mathrm{mM} \mathrm{NaCl}, 15 \mathrm{mM}$ imidazole, $0.2 \% \mathrm{NaN}_{3}$ (buffer A). The column was washed with $30 \mathrm{ml}$ of buffer $\mathrm{A}, 40 \mathrm{ml}$ of buffer $\mathrm{A}$ containing $7 \mathrm{M}$ guanidine hydrochloride, and $40 \mathrm{ml}$ of buffer $\mathrm{A}$. $\mathrm{A}$ solution $(7 \mathrm{ml}$ ) containing $2 \mathrm{mM}$ isotope-labeled $\mathrm{FMN}$ in buffer A was allowed to circulate through the column for 20 hours at $+4{ }^{\circ} \mathrm{C}$. The column was washed with buffer $\mathrm{A}$, and the protein was eluted by $50 \mathrm{mM}$ Tris hydrochloride, $\mathrm{pH} 8.0$, containing $400 \mathrm{mM} \mathrm{NaCl}, 120 \mathrm{mM}$ imidazole and $0.2 \% \mathrm{NaN}_{3}$ ). Fractions were concentrated and transferred into $40 \mathrm{mM}$ sodium/potassium phosphate, $\mathrm{pH}$ 7.0, containing $0.2 \% \mathrm{NaN}_{3}$ ) by ultrafiltration. They were stored at $-80^{\circ} \mathrm{C}$.

\section{Results and Discussion}

Figure 2 and Table 1 present the principal experimental and computational results of this paper. Figure 2 a shows the isoalloxazine chromophore including the atom numbering scheme used. Figure $2 c$ and e present experimental FSRS spectra from FMN and four isotopologues, measured 2 ps and 3 ns after electronic excitation of FMN at $450 \mathrm{~nm}$; for FMN (or FAD) itself there is good agreement with the experimental data presented here and those of Weigel et al and Andrikopoulos et al. The 2 ps data reflect the FSRS spectrum of the $S_{1}$ excited electronic state, and were previously shown to 
not evolve on the timescale of tens of picoseconds. ${ }^{22}$ The excited singlet state lifetime of FMN is 4 ns and its decay is mainly to the triplet state, $T_{1}$ (via intersystem crossing with a quantum yield of the order of 0.2 to 0.6$).^{45-47}$ The temporal evolution of the FSRS spectrum is assigned to formation of the $T_{1}$ state. The $S_{1}$ lifetime is longer than the accessible delay time for the delay stage used. However, we find that the FSRS spectrum does not evolve further beyond $2 \mathrm{~ns}$, but that the FSRS signal amplitude increases between tens of picoseconds and $3 \mathrm{~ns}$; these data are shown in SI5. This occurs as a result of intersystem crossing (as previously seen in resonant $\mathrm{FSRS}^{44}$ ). This increased amplitude reflects the stronger resonance enhancement of $T_{1}$ at the $750 \mathrm{~nm}^{23}$ Raman pump wavelength used, when compared to the singlet state (recalling that FSRS signal scales as the fourth power of the transition moment ${ }^{48}$ ); this is also evident in the enhanced signal to noise in the later time spectra (Figure 2). We thus conclude that data recorded at $3 \mathrm{~ns}$ represent FSRS of the $\mathrm{T}_{1}$ state. Note that the So Raman spectra are not presented here, as the focus is on the excited states; the ground state has been studied and assigned elsewhere. ${ }^{49-50}$ 
(a) Labelled Chromophore

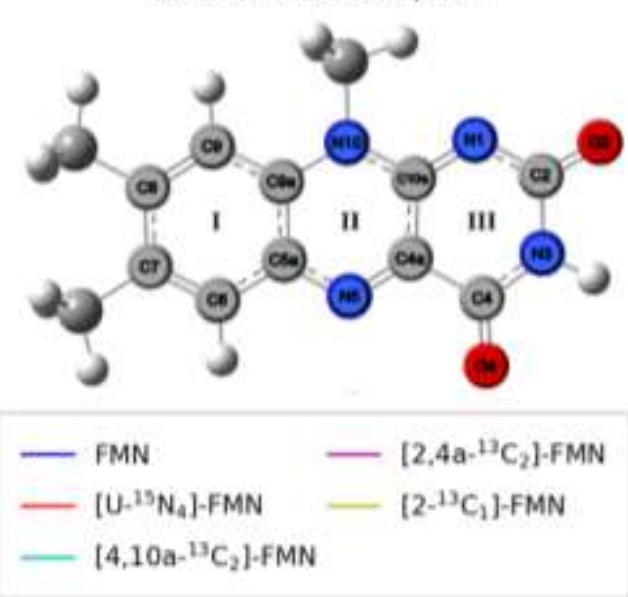

(c) Experimental $S_{1}$ FSRS

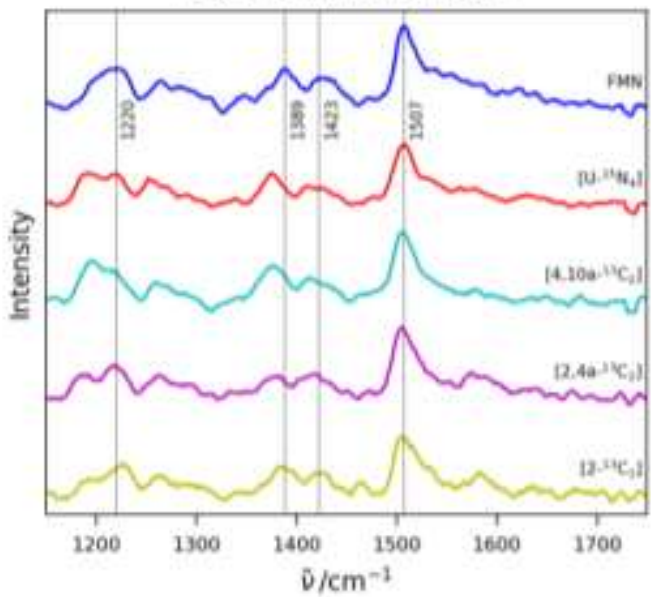

(e) Experimental $T_{1}$ FSRS

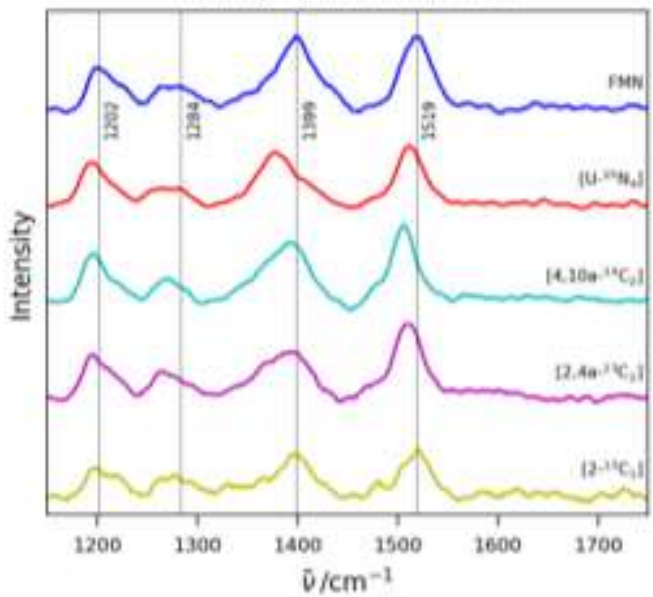

(b) Calculated S, RAMAN

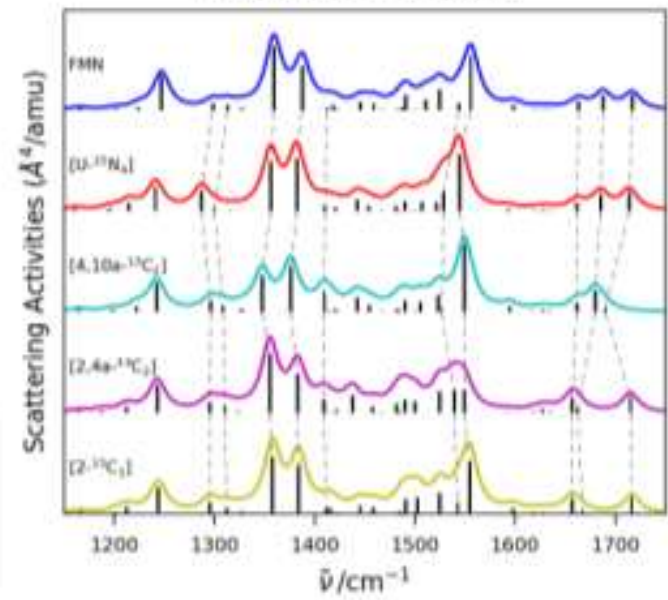

(d) Calculated $S_{1}$ RAMAN

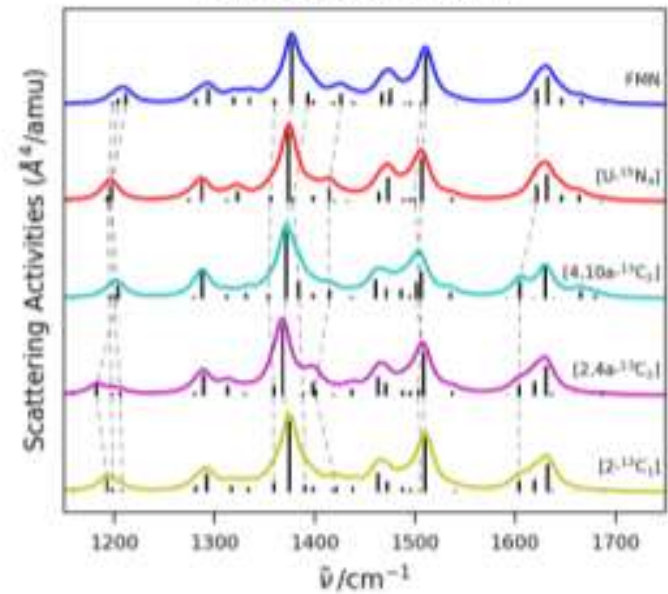

(f) Calculated $T_{2}$ RAMAN

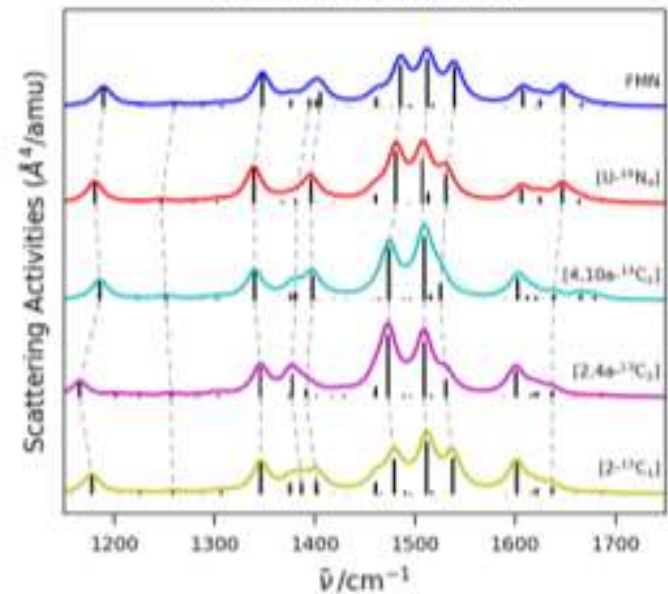

Figure 2: (a) Lumiflavin with atom designations. (b), (d) and (f), calculated Raman spectra for lumiflavin with 4 hydrogen-bonded water molecules; (b) $S_{0}$, (d) $S_{1},(f) T_{1}$. (c) and (e), FSRS spectra of FMN in $20 \mathrm{mM}$ Tris hydrochloride, $\mathrm{pH} 8.0$, containing $150 \mathrm{mM} \mathrm{NaCl}$; (c) $\mathrm{S}_{1}$, (e) $T_{1}$, arbitrarily offset for clarity. The calculated modes listed in Table 1 are indicated by dashed lines. The colour code for isotopologues is shown in (a). Additional calculated spectra for $\left[4 a_{-13}{ }^{13} C_{1}\right]-F M N$ are included in supporting information.

Figure $2 \mathrm{~b}, \mathrm{~d}, \mathrm{f}$ present the results of DFT $\left(\mathrm{S}_{0}, \mathrm{~T}_{1}\right)$ and TD-DFT $\left(\mathrm{S}_{1}\right)$ calculations of the Raman spectra for the H-bonded isoalloxazine chromophore shown in Figure 1a. Each shows the calculated 'stick' 
spectrum as well as a broadened spectrum, to allow for better comparison with experiment. The broadened spectra are obtained by applying a Lorentzian function with FWHM of $20 \mathrm{~cm}^{-1}$ (the estimated bandwidth of our spectrometer) to each 'stick' which are then scaled by an arbitrary constant such that the original 'stick' spectrum appears within the lineshape. In Figure $2 b-f$ we present in each case the measurements or calculations for FMN and the isotopologues studied: [U$\left.{ }^{15} \mathrm{~N}_{4}\right]-\mathrm{FMN} ;\left[4,10 \mathrm{a}-{ }^{13} \mathrm{C}_{2}\right]-\mathrm{FMN} ;\left[2,4 \mathrm{a}-{ }^{13} \mathrm{C}_{2}\right]-\mathrm{FMN} ;\left[2-{ }^{13} \mathrm{C}_{1}\right]-\mathrm{FMN}$. Additional calculated spectra for [4a$\left.{ }^{13} C_{1}\right]$-FMN are included in the supporting information. The calculations were performed for lumiflavin rather than FMN itself for both computational simplicity and relevance. While FMN has a ribityl plus phosphate side chain (which is absent in isoalloxazine and replaced by a methyl group in lumiflavin), that chain is not expected to contribute to the observed Raman spectrum, which is assumed to be dominated by the electronically resonant isoalloxazine chromophore; a consequence of this assumption is clear that ribityl chain modes may be missed. However, omitting the chain affords some other advantages. In particular, it is then not necessary to select a chain conformation, which, as Andrikopoulos et al. have shown, ${ }^{23}$ alters the calculated Raman spectra; in the actual solution at room temperature multiple interconverting conformers will be populated. Further, the presence of sidechain/chromophore interactions would also make the comparison with protein FSRS data more complex; in AsLOV2 the chain adopts an extended conformation, rather than folding back to interact with the chromophore, and should thus not be included in comparisons with experimental data. ${ }^{51}$

Before considering the isotope shifts of the individual modes, it is instructive to qualitatively compare measured and simulated data, where some clear similarities and important differences are apparent. Vibrational bands (or clusters of modes) are both observed and calculated near 1200 , 1400 and $1500 \mathrm{~cm}^{-1}$. In contrast, the cluster of Raman active modes calculated to appear above $1600 \mathrm{~cm}^{-1}$, which are mainly associated with the carbonyl stretches $(C 2=0$ and $C 4=0)$ are very weak or absent in the measurements. Here we recall that Figures $2 c$,e are recorded under conditions of resonance enhancement, where particularly strong $T_{1} \rightarrow T_{n}$ resonance is found in the region of the 
$750 \mathrm{~nm}$ pump, as well as a cluster of singlet states corresponding to $S_{1} \rightarrow S_{n}$ excited state absorption. ${ }^{23}$ While the observed frequencies represent vibrations of the initial state $\left(S_{1}\right.$ or $\left.T_{1}\right)$, the resonance Raman intensities depend upon the gradient of the upper $\left(S_{n}, T_{n}\right)$ state potential along the vibrational coordinate. ${ }^{48}$ These enhancement factors are not taken into account in the calculations of the off resonant Raman spectra. Thus it seems likely that the already modest intensity in the $\mathrm{C}=\mathrm{O}$ stretch modes do not gain from resonance enhancement, presumably because they are less displaced on electronic excitation than the ring modes for example, and are therefore very weak in the experimental spectra.

To assign the modes observed in the experimental spectra we compare the isotope shifts seen in the isotopologues studies with the calculated data. Clearly it is often the case that more than one calculated mode may contribute to any observed experimental band (Figure 2). We have identified the calculated modes which are most sensitive to isotope substitution, finding eight to ten modes in each electronic state (although these isotope sensitive modes are not the same ones in all three states). The modes are identified using the mode numbers generated from the Gaussian calculation for the case of the unlabelled isoalloxazine, specific to each electronic state $\left(\mathrm{S}_{0}, \mathrm{~S}_{1}\right.$ and $\left.\mathrm{T}_{1}\right)$. These selected modes are tracked through the different isotope labelled FMNs (dashed lines in Figure 2 $b, d, f)$. Any modes mainly localised on explicit waters are not considered, as in solution these will be dynamic and rapidly exchanging. As expected for any large molecule, the actual nuclear displacements in a given normal vibrational mode are quite complex and involve a number of bond stretches and bends. In Table 1 the main nuclear displacements that are calculated to contribute are listed, along with the corresponding wavenumber or isotope shift; those displacements that involve the isotopically edited atoms are shown in bold. Atom displacements for these modes are illustrated in the supplementary information. 


\begin{tabular}{|c|c|c|c|c|c|c|c|c|}
\hline Mode & $\begin{array}{l}\mathrm{FMN} \\
/ \mathrm{cm}^{-1}\end{array}$ & $\begin{array}{c}{\left[\mathrm{U}-{ }^{15} \mathrm{~N}_{4}\right]-\mathrm{FMN}} \\
/ \mathrm{cm}^{-1}\end{array}$ & $\begin{array}{c}{\left[4,10 \mathrm{a}-{ }^{13} \mathrm{C}_{2}\right]-\mathrm{FMN}} \\
/ \mathrm{cm}^{-1}\end{array}$ & $\begin{array}{c}{\left[2,4 a-{ }^{13} C_{2}\right]-F M N} \\
/ \mathrm{cm}^{-1}\end{array}$ & $\begin{array}{c}{\left[2-{ }^{13} \mathrm{C}_{1}\right]-\mathrm{FMN}} \\
/ \mathrm{cm}^{-1}\end{array}$ & $\begin{array}{l}\text { [N3D]-FMN } \\
/ \mathrm{cm}^{-1}\end{array}$ & $\begin{array}{c}{\left[\mathrm{N} 3 \mathrm{D}+\mathrm{D}_{2} \mathrm{O}\right]-\mathrm{FMN}} \\
/ \mathrm{cm}^{-1}\end{array}$ & Assignment ${ }^{i}$ \\
\hline \multicolumn{9}{|c|}{$S_{1}$ FSRS } \\
\hline & 1220 & +2 & -1 & -2 & +7 & & 0 & $74 / 75$ \\
\hline & 1389 & -14 & -13 & -8 & -2 & & -2 & 82 \\
\hline & 1423 & -13 & -9 & -5 & -1 & & +7 & 85 \\
\hline & 1507 & +1 & -1 & -1 & 0 & & +1 & 95 \\
\hline \multicolumn{9}{|c|}{$\mathrm{T}_{1}$ FSRS } \\
\hline & 1202 & -8 & -6 & -5 & -5 & & & 73 \\
\hline & 1284 & -2 & -12 & -20 & -5 & & -16 & \\
\hline & 1399 & -21 & -5 & -3 & 0 & & 0 & \\
\hline & 1519 & -8 & -11 & -9 & +3 & & -1 & $90 / 96$ \\
\hline \multicolumn{8}{|c|}{$S_{1}$ Calculated } & \\
\hline 73 & 1198 & -4 & -3 & 0 & +1 & & & sN5-C5a, sN3-C4, sC6-C7, wC6-H, wC9-H \\
\hline 74 & 1204 & -7 & 0 & -21 & -11 & -4 & -6 & $\begin{array}{c}\text { sC2-N3, ssN5-C4a-C10a, sC9-C9a, sC6-C7, wC6-H, } \\
w C 9-\mathrm{H}, w \mathrm{~N} 10-\mathrm{Me}\end{array}$ \\
\hline \multirow[t]{2}{*}{75} & 1212 & -16 & -12 & -6 & -4 & -10 & -11 & asC10a-N1-C2, sN3-C4, sC4a-N5, sC6-C7 \\
\hline & & & & & & +56 & +55 & \\
\hline 80 & 1360 & -4 & -6 & -1 & 0 & 0 & 0 & $\begin{array}{c}\text { sN10-C10a, sN3-C4, asC7-C8-C9, asC6-C5a-C9a, } \\
\text { bN10-Me, bC7-Me, bC8-Me }\end{array}$ \\
\hline 81 & 1377 & -3 & -5 & -9 & -2 & +2 & +2 & sC4a-C10a, sN1-C2, sC5a-C9a, sC6-C7, wC6-H \\
\hline 82 & 1393 & -14 & -9 & -4 & -2 & 0 & +1 & $\begin{array}{c}s \mathrm{C} 4 \mathrm{a}-\mathrm{N} 5, \boldsymbol{s N 1 0 - C 1 0 a}, \boldsymbol{s N 1 - C 2}, s s \mathrm{C} 8-\mathrm{C9}-\mathrm{C} 9 \mathrm{a}, w C 6-\mathrm{H}, \\
b \mathrm{~N} 10-\mathrm{Me}, w C 8-\mathrm{Me}, w C 7-\mathrm{Me}\end{array}$ \\
\hline 85 & 1426 & -11 & -11 & -25 & -4 & -1 & -1 & $\begin{array}{c}\text { asN5-C4a-C4, ssC10a-N1-C2, sN3-C4, wC6-H, } \\
\text { scC8-Me, scN10-Me }\end{array}$ \\
\hline 94 & 1506 & -6 & 0 & -2 & -1 & -4 & -4 & $\begin{array}{c}\text { sC9a-N10, ssN5-C4a-C4, sN1-C10a, sC6-C7, wN3-H, } \\
b \text { C7-Me, bC8-Me, bN10-Me }\end{array}$ \\
\hline 95 & 1511 & -4 & -9 & -2 & 0 & -2 & -2 & $\begin{array}{c}\text { sC4a-N5, sN1-C10a, sC7-C8, sC5a-C9a, wN3-H, } \\
b \mathrm{C} 7-\mathrm{Me}, b \mathrm{C} 8-\mathrm{Me}, b \mathrm{~N} 10-\mathrm{Me}, w C 9-\mathrm{H}, w \mathrm{C} 6-\mathrm{H}\end{array}$ \\
\hline 99 & 1622 & 0 & -17 & -18 & -18 & -1 & & $s s(\mathrm{C} 2=02, \mathrm{C} 4=04), w N 3-\mathrm{H}, b \mathrm{H}_{2} \mathrm{O}$ \\
\hline
\end{tabular}




\begin{tabular}{|c|c|c|c|c|c|c|c|c|}
\hline \multicolumn{8}{|c|}{$\mathrm{T}_{1}$ Calculated } & \\
\hline 73 & 1190 & -9 & -4 & -24 & -12 & +1 & -6 & $\begin{array}{c}\text { asN1-C2-N3, asC4-C4a-C10a, asC5a-C6-C7, wC6-H, } \\
\text { ssC8-C9-C9a }\end{array}$ \\
\hline 76 & 1260 & -13 & -8 & -3 & -1 & & & $\begin{array}{c}\text { asC2-N3-C4, sN10-C10a, sC4a-N5, sC6-C7, wC6-H, } \\
w \mathrm{~N} 10-\mathrm{Me}\end{array}$ \\
\hline 79 & 1348 & -8 & -8 & -2 & -2 & -2 & -2 & $\begin{array}{c}\text { sN10-Me, sN1-C10a, ssC2-N3-C4, asC6-C7-C8, } \\
\text { asC9-C9a-C5a, wC6-H, wC9-H }\end{array}$ \\
\hline 81 & 1395 & -14 & -14 & -17 & -8 & +2 & +3 & $\begin{array}{c}s \mathrm{~N} 1-\mathrm{C} 10 \mathrm{a}, \mathrm{sC4a-N5,} s s \mathrm{C2}-\mathrm{N3}-\mathrm{C} 4, s \mathrm{C} 9-\mathrm{C} 9 \mathrm{a}, b \mathrm{C7}-\mathrm{Me} \text {, } \\
w \mathrm{~N} 3-\mathrm{H}\end{array}$ \\
\hline 83 & 1406 & -10 & -8 & -14 & -3 & -1 & -1 & $\begin{array}{c}\text { sN10-C10a, sC4a-N5, sN1-C2, sN3-C4, ssC8-C9-C9a, } \\
\text { wC7-Me, wC8-Me, wN10-Me }\end{array}$ \\
\hline 90 & 1486 & -5 & -11 & -13 & -6 & & & $s \mathrm{C} 4 \mathrm{a}-\mathrm{N} 5, \mathrm{sN} 1-\mathrm{C} 10 \mathrm{a}, a s(\mathrm{C} 2=02, \mathrm{C} 4=04), w \mathrm{~N} 3-\mathrm{H}$ \\
\hline 94 & 1512 & -4 & -3 & -3 & 0 & -1 & 0 & $\begin{array}{c}\text { sC4a-N5, sN1-C10a, ssC5a-C6-C7, sC8-Me, } \\
\text { sC9a-N10, wN3-H, bN10-Me, bC7-Me }\end{array}$ \\
\hline 96 & 1540 & -9 & -14 & -9 & -2 & -10 & -9 & $\begin{array}{c}s \mathrm{C} 4 \mathrm{a}-\mathrm{N} 5, s \mathrm{~N} 1-\mathrm{C} 10 \mathrm{a}, \mathrm{sC2}=\mathrm{O2}, \mathrm{sN3}-\mathrm{C} 4, s s \mathrm{C} 7-\mathrm{C} 8-\mathrm{C} 9 \\
w \mathrm{C} 6-\mathrm{H}, w \mathrm{C} 9-\mathrm{H}, w \mathrm{~N} 3-\mathrm{H}\end{array}$ \\
\hline 101 & 1648 & 0 & -9 & -11 & -11 & -1 & -3 & $\begin{array}{c}s s(\mathrm{C} 2=02, \mathrm{C} 4=04), s \mathrm{C} 5 \mathrm{a}-\mathrm{C} 6, s \mathrm{C} 8-\mathrm{C} 9, s \mathrm{C} 10 \mathrm{a}-\mathrm{N} 10 \\
w C 6-\mathrm{H}, w C 9-\mathrm{H}\end{array}$ \\
\hline \multicolumn{8}{|c|}{ So Calculated } & \\
\hline 76 & 1299 & -11 & -2 & -3 & -3 & & & $\begin{array}{c}\text { sN1-C2, sN5-C5a, sN10-C10a, sN3-C4, wC6-H, } \\
w C 9-\mathrm{H}\end{array}$ \\
\hline 77 & 1313 & -13 & -4 & -2 & 0 & & & $\begin{array}{l}\text { sN3-C4, ssN10-C10a-N1, sC4a-N5, sC5a-C6, } \\
\text { asC7-C8-C9, wC6-H, wC9-H, wN10-Me }\end{array}$ \\
\hline 79 & 1359 & -3 & -11 & -3 & -1 & & & $\begin{array}{c}\text { as N10-C10a-C4a, ssC2-N3-C4, asC5a-C9a-C9, } \\
s \mathrm{C} 7-\mathrm{C} 8, w \mathrm{C} 6-\mathrm{H}\end{array}$ \\
\hline 80 & 1388 & -6 & -12 & -5 & -4 & & & $\begin{array}{c}\text { ssC10a-N1-C2, asC4a-C4-N3, sC5a-C9a, sC8-C9, } \\
\text { scN10-Me }\end{array}$ \\
\hline 81 & 1413 & -2 & -3 & -4 & -1 & & & sC4a-C4, sN1-C2, ssC5a-C6-C7, scC7-Me, scC8-Me \\
\hline 94 & 1544 & -15 & -17 & -5 & -1 & & & $\begin{array}{c}\text { sN1-C10a, sC4a-N5, sN3-C4, sC9-C9a, sC7-C8, } \\
w N 3-\mathrm{H}, \text { as }(\mathrm{C} 2=\mathrm{O} 2, \mathrm{C} 4=\mathrm{O} 4)\end{array}$ \\
\hline 95 & 1556 & -11 & -6 & -6 & -1 & & & $\begin{array}{c}\text { asN10-C10a-N1, sC4a-N5, ssC8-C9-C9a, ss(C2=O2, } \\
(\mathrm{C} 4=\mathrm{O} 4), w \mathrm{~N} 3-\mathrm{H}, b \mathrm{~N} 10-\mathrm{Me}\end{array}$ \\
\hline
\end{tabular}




\begin{tabular}{|c|c|c|c|c|c|c|}
\hline 101 & 1663 & -1 & -1 & -6 & -6 & $s \mathrm{C} 2=02, s \mathrm{C} 4 \mathrm{a}-\mathrm{N} 5, s \mathrm{C} 6-\mathrm{C} 7, s \mathrm{C} 9-\mathrm{C} 9 \mathrm{a}$ \\
\hline 102 & 1688 & -3 & -8 & -25 & -21 & $s \mathrm{C} 2=02, w N 3-\mathrm{H}, b \mathrm{H}_{2} \mathrm{O}$ \\
\hline 103 & 1717 & -3 & -27 & -2 & 0 & $s \mathrm{C} 4=04, w \mathrm{~N} 3-\mathrm{H}, b \mathrm{H}_{2} \mathrm{O}$ \\
\hline
\end{tabular}

Table 1: Experimental FSRS peaks and relevant calculated modes of FMN in states $S_{1}, T_{1}$ and $S_{0}$ with corresponding frequency shifts for all isotopologues shown in Figure 2 and on deuteration of FMN in states $S_{1}$ and $T_{1}$ shown in Figure 3. Calculated modes are numbered according to the Gaussian output for each electronic state and assigned in terms of the main nuclear displacements, where stretches involving isotopically substituted atoms are shown in bold. s: stretch, a-: antisymmetric, s-: symmetric, w: wag, t: twist, sc: scissor, $r$ : rock, $b$ : bend. Three atom stretches are described with respect to the centre atom and delocalised/coupled carbonyl stretches are indicated using brackets. ${ }^{i}$ Mode numbers given for FSRS assignments refer to the calculations presented below, as discussed in the text. 
4 S1 Raman Assignments. Concerning the experimental spectra (Figure 2c), we note that there are

5 indications of Raman mode activity above $1600 \mathrm{~cm}^{-1}$ which might be associated with $\mathrm{C}=\mathrm{O}$ modes, but this is so weak that we do not attempt a more definitive assignment. The next lower wavenumber band clearly observed is at $1507 \mathrm{~cm}^{-1}$ in FMN. This band has the strongest observed activity and is insensitive to all isotope exchange patterns studied. Continuing to lower wavenumber, a pair of bands are measured at 1423 and $1389 \mathrm{~cm}^{-1}$, with the lower wavenumber contribution being particularly susceptible to isotopic substitution (Figure 2c). The lowest wavenumber band considered here is a broad asymmetric band at $1220 \mathrm{~cm}^{-1}$ in FMN. This is resolved into a doublet in all of the isotopes studied, with characteristic patterns for each isotopologue.

Turning to the calculated data, it is interesting that the cluster of modes above $1600 \mathrm{~cm}^{-1}$ involving the $\mathrm{C}=\mathrm{O}$ stretching modes are at lower wavenumber than in $\mathrm{S}_{0}$ (Figure $2 \mathrm{~b}, \mathrm{~d}$ ) and have additional modes contributing, suggesting these bonds are weakened on $\pi \pi^{*}$ excitation. The only major isotope shifts are for mode 99 , the symmetric $\mathrm{C} 2=\mathrm{O} / \mathrm{C} 4=0$ stretch, which is evidently (and not unexpectedly) red shifted by $\mathrm{C} 2$ and $\mathrm{C} 4{ }^{13} \mathrm{C}$ exchange. To lower wavenumber, the most intense mode calculated near the observed $1507 \mathrm{~cm}^{-1}$ band in FMN is found at $1511 \mathrm{~cm}^{-1}$ (mode 95). In four of the isotopologues investigated this mode shifts by less than $4 \mathrm{~cm}^{-1}$, consistent with the experimental observations. This mode involves a number of $\mathrm{CC}$ and $\mathrm{CN}$ ring stretches. Interestingly a $9 \mathrm{~cm}^{-1}$ red shift is calculated for $\left[4,10 a-{ }^{-13} C_{2}\right]-F M N$, which is not observed experimentally. However, in this particular isotopologue, mode 95 decreases in amplitude and its wavenumber crosses below that of mode 94. Mode 94 undergoes a corresponding increase in its amplitude, from very weak to strong; this result is thus consistent with the experimentally observed isotope insensitivity in $\left[4,10 a-{ }^{13} C_{2}\right]-$ FMN.

There are moderately intense modes calculated at 1475 and $1467 \mathrm{~cm}^{-1}$, which are absent in the experimental spectra, perhaps because they do not benefit from resonance enhancement (these modes are more localised on the methyl groups - see supporting information). The next cluster of modes includes the most intense, at $1377 \mathrm{~cm}^{-1}$ in FMN. This cluster must contribute to the pair of 
bands observed at 1423 and $1389 \mathrm{~cm}^{-1}$; with regards to isotope effects mode 85 and $80,81,82$ respectively appear to be the major contributors to the observed Raman shifts. Mode 85 at 1426 $\mathrm{cm}^{-1}$ in FMN has contributions from stretches involving atoms N5, C4a, C4, C10a, N1 and C2, consistent with its strong calculated isotope dependence; C4a exchange has a particularly marked effect, which aligns with the experimental result for $\left[2,4 a-{ }^{13} C_{2}\right]-F M N$ (Figure $\left.2 c\right)$. For the lower wavenumber contribution (modes $80,81,82$ which involve $C C$ and $C N$ stretches spread over all three rings, see Table 1 and supporting information) the calculated shift between $\mathrm{FMN}$ and $\left[2-{ }^{13} \mathrm{C}_{1}\right]-\mathrm{FMN}$ is $<2 \mathrm{~cm}^{-1}$, consistent with measurement. For $\left[\mathrm{U}-{ }^{15} \mathrm{~N}_{4}\right]-\mathrm{FMN},\left[4,10 \mathrm{a}-{ }^{13} \mathrm{C}_{2}\right]-\mathrm{FMN}$ and $\left[2,4 \mathrm{a}-{ }^{13} \mathrm{C}_{2}\right]-\mathrm{FMN}$ red shifts are both calculated and observed.

The pair of observed modes derived from the single broad $1220 \mathrm{~cm}^{-1}$ signal in FMN have contributions from modes 73,74 and 75 . In particular mode 75 contributes to the downshift of the lower wavenumber component in $\left[\mathrm{U}-{ }^{15} \mathrm{~N}_{4}\right]-\mathrm{FMN}$ and $\left[4,10 \mathrm{a}-{ }^{13} \mathrm{C}_{2}\right]-\mathrm{FMN}$, while mode 74 plays a similar role for $\left[2,4 a_{-13}{ }^{13} C_{2}\right]-F M N$ and $\left[2-{ }^{13} C_{1}\right]-F M N$. Modes 74 and 75 have contributions from ring stretching in all three rings, while the smaller isotope shifts in mode 3 reflect its greater localisation on ring I.

Summarising, for the four bands clearly observed in the FSRS spectra of $S_{1} F M N$, we make the following assignments. The doublet character of $1220 \mathrm{~cm}^{-1}$ on isotope substitution suggests at least the involvement of modes 74 and 75 . These mainly involve framework stretch modes spread over rings $\mathrm{I}-\mathrm{III}$, without involvement of the $\mathrm{N} 3 \mathrm{H}$ wag. For the experimental $1389 \mathrm{~cm}^{-1}$ band, modes 80,81 , 82 can contribute, and the isotope shifts observed point to mode 82 . This mode is characterised by $\mathrm{CN}$ ring stretches and $\mathrm{C} 6 \mathrm{H}$ wag. The $1423 \mathrm{~cm}^{-1}$ band is tentatively ascribed to mode 85 , although it lacks the large shift calculated for $\left[2,4 a-{ }^{13} \mathrm{C} 2\right]-\mathrm{FMN}$. Mode 85 mainly comprises $\mathrm{CN}$ stretches in ring III and methyl wag motions. Finally, the negligible isotope effect in the intense $1507 \mathrm{~cm}^{-1}$ band is best represented in mode 95 (although the assignment required the calculated change in character to mode 94 for different isotopologues to be considered, as described above). Again that mode involves $\mathrm{CN}$ and $\mathrm{CC}$ ring stretches as well as $\mathrm{N} 3 \mathrm{H}$ and methyl wag. 
Comparing to previous literature is difficult, as some assignments involve calculated modes involving the ribityl chain, which is not included in our calculation. Indeed, Andrikopoulos et al. include two specific sidechain conformations with differing assignments. ${ }^{23}$ Further, Weigel et al.'s discussion of $\mathrm{S}_{1}$ assignments is mainly focused on the deuteration effects. ${ }^{21}$ Nevertheless, some comparisons are possible. The intense high frequency band observed here at $1507 \mathrm{~cm}^{-1}$ aligns with $1505 \mathrm{~cm}^{-1}$ band of Weigel et al. In the absence of isotope shifts a number of their calculated modes were possible assignments, each mainly involving ring stretches, consistent with our assignment of mode 95. Andrikopoulos et al. assign the band at $1500 \mathrm{~cm}^{-1}$ band to a higher frequency mode involving CO stretch, N3H wag and explicit water bending, but also discuss an improved assignment to an alternative mode comprised of ring stretches, in alignment with the present results. Our $1423 \mathrm{~cm}^{-1}$ band aligns with the $1421 \mathrm{~cm}^{-1}$ band of Weigel et al. Again they have multiple possibilities, mainly involving ring modes. Andrikopoulos et al. suggest either ring modes or $\mathrm{CH}$ twist/rock may be important, depending on the specific conformation of the ribityl chain. The present data support an assignment to ring modes. Our $1389 \mathrm{~cm}^{-1}$ band compares with the $1387 \mathrm{~cm}^{-1}$ of Weigel et al, with both assignments involving ring displacements. Andrikopoulos et al. report a $1384 \mathrm{~cm}^{-1}$ band, again mainly assigned to $\mathrm{CH}$ motion, which we do not detect as a major contribution, although our calculation has methyl rather than the ribityl chain. In all three studies the bands between 1200 $1260 \mathrm{~cm}^{-1}$ are multiplet, making further comparison challenging.

$\mathbf{T}_{\mathbf{1}}$ Raman Assignments. For the observed $\mathrm{T}_{1}$ spectra there are four well resolved but asymmetric bands (Figure 2e). There is no measurable activity resolved above $1550 \mathrm{~cm}^{-1}$, consistent with the negligible contribution from the CO modes in the resonant FSRS. The highest wavenumber band observed is at $1519 \mathrm{~cm}^{-1}$. This is sensitive to isotope substitution, in contrast to the highest wavenumber band in the $S_{1}$ spectrum $\left(1507 \mathrm{~cm}^{-1}\right)$ indicating that these two bands are of different origin. In order of decreasing wavenumber, the next band at $1399 \mathrm{~cm}^{-1}$ in FMN is insensitive to isotope substitution except in the case of $\left[\mathrm{U}^{15} \mathrm{~N}_{4}\right]-\mathrm{FMN}$, where a red shift is observed. Next is the 
band at $1284 \mathrm{~cm}^{-1}$ in FMN which shows significant isotope shifts of up to $20 \mathrm{~cm}^{-1}$, while the final band is at $1202 \mathrm{~cm}^{-1}$ (FMN) and has a weaker red shift of $8 \mathrm{~cm}^{-1}$ at most.

Considering the carbonyl region $\left(>1550 \mathrm{~cm}^{-1}\right)$ calculated for $T_{1}$, we note again the relatively large number of modes which contribute in the excited state, compared to the ground state, and also that the mean wavenumber of this cluster of modes has shifted slightly further to the red in $T_{1}$ compared to $S_{1}$. At lower wavenumber, the experimentally observed relatively narrow band at $1519 \mathrm{~cm}^{-1}$ (FMN) corresponds to a cluster of intense modes in the calculation between 1480 and $1550 \mathrm{~cm}^{-1}$. The three strongest are modes 90, 94, 96 (see Table 1). From these, 90 and 96 exhibit shifts of between 5 and $14 \mathrm{~cm}^{-1}$ in $\left[\mathrm{U}^{-15} \mathrm{~N}_{4}\right]-\mathrm{FMN},\left[4,10 \mathrm{a}-{ }^{13} \mathrm{C}_{2}\right]-\mathrm{FMN}$ and $\left[2,4 \mathrm{a}_{-}{ }^{13} \mathrm{C}_{2}\right]-\mathrm{FMN}$, which are on the same scale as the experimental shifts observed. A shift of $\leq 6 \mathrm{~cm}^{-1}$ is seen for $\left[2-{ }^{13} C_{1}\right]-F M N$, again consistent with experiment. Mode 96 has its dominant contributions for ring stretch modes spread over all three rings, while mode 90 is localised on rings II and III. Mode 94 is more localised on ring I, consistent with its smaller isotope shifts.

The broad band observed at $1399 \mathrm{~cm}^{-1}$ in FMN corresponds with calculated modes 79, 81, 83 (1348, 1395 and $1406 \mathrm{~cm}^{-1}$ in FMN). The most significant $\left(20 \mathrm{~cm}^{-1}\right)$ isotope red-shift observed was for [U${ }^{15} \mathrm{~N}_{4}$ ]-FMN, where there is also a marked change in the asymmetry of the band. The peak shift is larger than the largest calculated isotope shift $\left(17 \mathrm{~cm}^{-1}\right.$ for $\left.\left[2,4 a^{-13} C_{2}\right]-F M N\right)$. Thus to account for the large shift in $\left[\mathrm{U}^{-15} \mathrm{~N}_{4}\right]-\mathrm{FMN}$ it seems likely that the isotope red-shift is accompanied by a change in the dominant character of this mode, i.e. the main mode contributing in $\left[\mathrm{U}-{ }^{15} \mathrm{~N}_{4}\right]-\mathrm{FMN}$ is distinct from that in FMN. Indeed, the calculations indicate that the Raman activity changes between these three modes depending on the pattern of isotope substitution (Figure 2f).

The next band, observed at $1284 \mathrm{~cm}^{-1}$ in FMN, occurs in a region which is rather quiet in the calculated spectrum. We have identified the very weak mode 76 , dominated by NC stretches, as a possible candidate for resonant enhancement, but the isotope shifts calculated for this mode are weaker than those observed. The final experimental band is at $1202 \mathrm{~cm}^{-1}$ in FMN. The only potential 
assignment from the calculations is mode 73 at $1190 \mathrm{~cm}^{-1}$, which has delocalised CN and CC stretch contributions. However, the calculations do not reproduce the rather modest isotope shifts observed, in particular a large shift is predicted for $\left[2,4 a-{ }^{13} C_{2}\right]-F M N$ which is not seen experimentally. Summarising, the $1519 \mathrm{~cm}^{-1}$ band is assigned to one of either modes 90 or 96 , both of which have prominent $\mathrm{CN}$ and $\mathrm{CO}$ stretch contributions, as well as $\mathrm{N} 3 \mathrm{H}$ wag. Thus, the mode character is indeed different to the strong $1507 \mathrm{~cm}^{-1}$ mode of the $S_{1}$ state. This assignment accords with that of Andrikopoulos et al. for one of their conformations. ${ }^{23}$ Neither $1399 \mathrm{~cm}^{-1}$ nor $1284 \mathrm{~cm}^{-1}$ bands are readily assigned based on the current calculations, and we suspect resonance enhancements must play an important role. The likely assignment of the $1202 \mathrm{~cm}^{-1}$ band is mode 73 , which again involves CN stretch modes.

$S_{0}$ Raman Assignments. The focus of this paper is on the $S_{1}$ and $T_{1}$ states, but we conclude with comments on the $S_{0}$ state calculations. This state has been investigated in detail by a number of groups, including studies of some of the isotopologues investigated here. Many of the most isotope sensitive modes mainly comprise ring stretching, and are thus consistent with the large isotope shifts associated with $\left[\mathrm{U}^{15} \mathrm{~N}_{4}\right]-\mathrm{FMN}$ and $\left[4,10 \mathrm{a}-{ }^{13} \mathrm{C}_{2}\right]-\mathrm{FMN}$. An interesting observation concerns the large shifts found in higher wavenumber modes associated with $\mathrm{C}=\mathrm{O}$ stretches. The highest wavenumber mode (mode 103) in FMN is a C4=O localised stretch with N3H wag (in-plane bend). However, specifically in $\left[4,10 a-{ }^{13} C_{2}\right]-F M N$ this mode develops a delocalised $C 4=O / C 2=O$ antisymmetric stretch character accompanied by a $27 \mathrm{~cm}^{-1}$ red shift (see supporting information, where relevant nuclear displacements are indicated). Mode 102 is primarily $\mathrm{C} 2=\mathrm{O}$ with $\mathrm{N} 3 \mathrm{H}$ wag in $\mathrm{FMN}$, but in $\left[4,10 a-{ }^{13} \mathrm{C}_{2}\right]-$ FMN the character of the mode is again delocalised, now as the symmetric stretch of the carbonyls. However, this leads to only an $8 \mathrm{~cm}^{-1}$ shift, the largest isotope shift being observed for $\left[2,4 a-{ }^{13} \mathrm{C}_{2}\right]-$ FMN $\left(25 \mathrm{~cm}^{-1}\right)$ with a slightly smaller shift in $\left[2-{ }^{13} \mathrm{C}_{1}\right]-\mathrm{FMN}$. The change in character of the $\mathrm{C}=\mathrm{O}$ stretches in FMN, which is accompanied by large spectral shifts, has been noted before on deuteration of $\mathrm{N} 3 \mathrm{H}$ (to which we return below). ${ }^{15-16}$ However, that the relatively smaller perturbation of ${ }^{12} \mathrm{C} /{ }^{13} \mathrm{C}$ exchange can have similar effects is significant, because wavenumber shifts 
in the carbonyl region of flavoproteins are often taken as indicative of specific $\mathrm{H}$-bonding interactions at either $\mathrm{C} 4=\mathrm{O}$ or $\mathrm{C} 2=0 .{ }^{52}$ It should always be borne in mind that these modes may become more (or less) delocalised under some circumstances, and that can also give rise to large spectral shifts; thus the support of isotope exchange and/or calculation is important in definitive assignments of carbonyl wavenumber shifts.

Effect $\mathrm{H}_{2} \mathrm{O} / \mathrm{D}_{2} \mathrm{O}$ Exchange The majority of transient IR data have been recorded in $\mathrm{D}_{2} \mathrm{O}$ to avoid the distorting effects of the strong absorbance of the $\mathrm{H}_{2} \mathrm{O}$ bending mode in the region of most interest. It has previously been shown that $\mathrm{H}_{2} \mathrm{O} / \mathrm{D}_{2} \mathrm{O}$ exchange causes significant changes in the IR spectra of isoalloxazine. Exchange of N3H for N3D was shown to result in a $13 \mathrm{~cm}^{-1}$ downshift in the $\mathrm{C} 4=\mathrm{O}$ mode. ${ }^{53}$ We also reported that this exchange (coupled with whether or not $\mathrm{H}$-bonds were formed) caused a complex variation in the character of the $\mathrm{C}=\mathrm{O}$ stretch $+\mathrm{N} 3 \mathrm{H}$ wag modes. ${ }^{15-16}$ Specifically, the $H / D$ exchange led to changes from two localised $C=O$ stretches to a coupled symmetric/antisymmetric pair of $\mathrm{C}=\mathrm{O}$ stretches, both accompanied by N3H/D wag. This change in character resulted in large spectral shifts; a similar change in character was calculated on ${ }^{13} \mathrm{C}$ substitution (Figure 2, above). It thus seems worthwhile to investigate the effect of $D_{2} \mathrm{O}$ on the excited state Raman spectra. This was previously considered for the $\mathrm{S}_{1}$ state FSRS by Ernsting and coworkers. $^{21}$

In Figure 3a and $\mathrm{c}$ the experimental FSRS data are presented for the $S_{1}$ and $T_{1}$ states of FMN respectively, measured in $\mathrm{H}_{2} \mathrm{O}$ and $\mathrm{D}_{2} \mathrm{O}$. The corresponding calculations are shown in Figures $3 \mathrm{~b}$ and $d$, but in this case we calculate first the effect of N3H/D exchange and then the result of exchanging all $\mathrm{H}$-bonded $\mathrm{H}_{2} \mathrm{O}$ to $\mathrm{D}_{2} \mathrm{O}$. The calculations show that both N3H/D exchange and an $\mathrm{H} / \mathrm{D}$-bonding environment affect the spectra in the carbonyl region, for both $S_{1}$ and $T_{1}$. This is in line with similar effects observed for IR data in the $S_{0}$ state. ${ }^{15-16}$ However, the weak Raman signal for these modes does not permit comparison with experiment. 
(a) Experimental $S_{1}$ FSRS

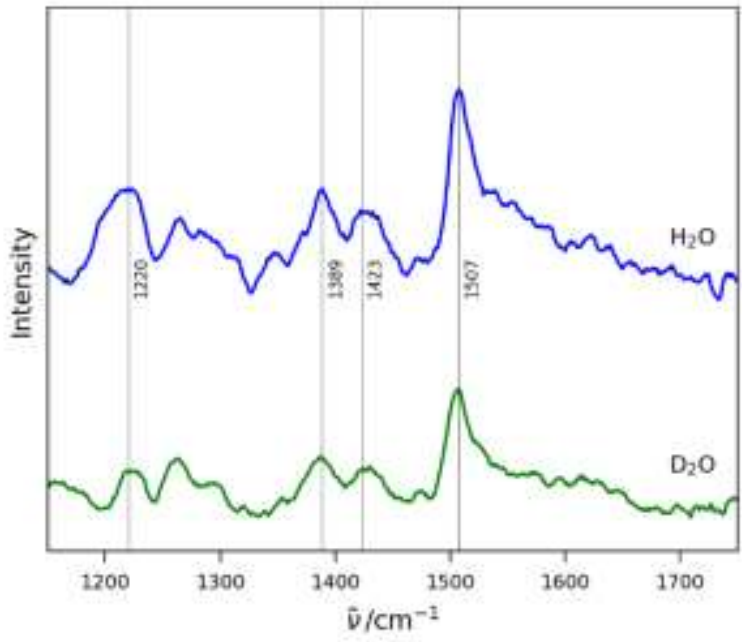

(c) Experimental T 1 FSRS



(b) Calculated $\mathrm{S}_{1}$ RAMAN

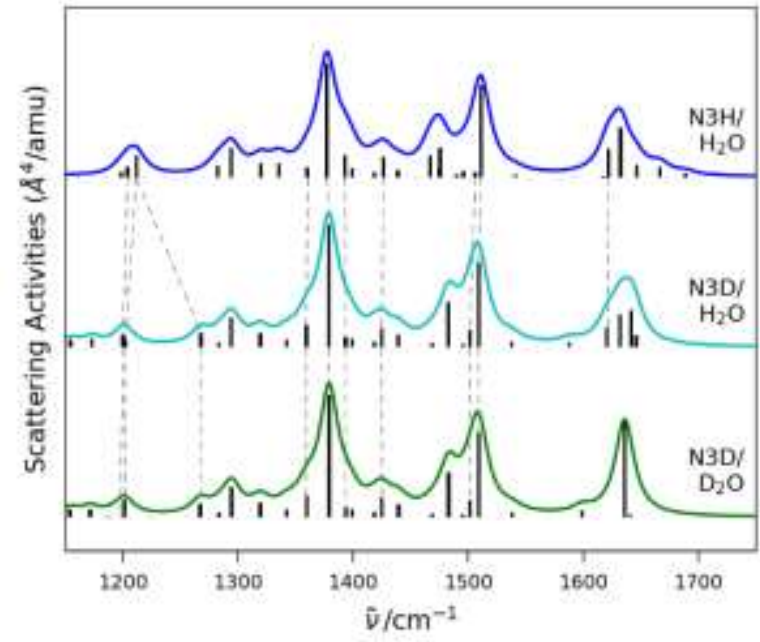

(d) Caiculated $T_{1}$ RAMAN

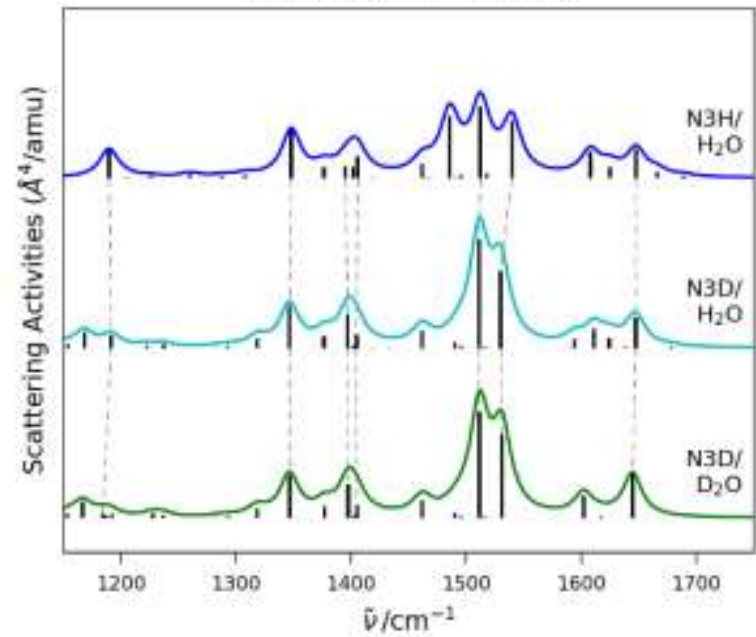

Figure 3: FSRS spectra of FMN in $\mathrm{H}_{2} \mathrm{O}$ buffer $(20 \mathrm{mM}$ Tris hydrochloride, $\mathrm{pH}$ 8.0, containing $150 \mathrm{mM} \mathrm{NaCl})$ (blue) and $\mathrm{D}_{2} \mathrm{O}$ (green), (a), $S_{1} ;(c), T_{1}$. Calculated Raman spectra of lumiflavin, (b) $S_{1}$ and (d) $T_{1}$. Spectra of FMN calculated with all $\mathrm{H}$ atoms (blue), exchange of $\mathrm{N} 3 \mathrm{H}$ to $\mathrm{N} 3 \mathrm{D}$ only (cyan) and full $\mathrm{N} 3 \mathrm{D}+\mathrm{D}_{2} \mathrm{O}$ substitution (green). The calculated modes listed in Table 1 are indicated by dashed lines.

For the $S_{1}$ excited state, the measured band at $1507 \mathrm{~cm}^{-1}$ is insensitive to $H / D$ exchange. The contributing modes were assigned as 94 and 95 (Table 1). These are dominated by ring stretches, but both do have a component of N3H wag (see supporting information). However, experiment and calculation agree that this does not yield significant sensitivity to N3H/D exchange (shifts of 4 and 2 $\mathrm{cm}^{-1}$ respectively, Figure 3a,b). Similarly, the 1389 and $1423 \mathrm{~cm}^{-1}$ bands are observed and calculated to be insensitive to either $\mathrm{N} 3 \mathrm{H}$ or $\mathrm{H}_{2} \mathrm{O}$ exchange. The band at $1220 \mathrm{~cm}^{-1}$ is much more sensitive to exchange (a similar effect having been noted by Weigel et al ${ }^{21}$ ). Calculations show that the dominant effect of $\mathrm{D}_{2} \mathrm{O}$ is due to $\mathrm{N} 3 \mathrm{H} / \mathrm{D}$ exchange rather than the $\mathrm{H}$-bond environment (Figure $3 \mathrm{~b}$ ). The result 
of exchange is that the $1220 \mathrm{~cm}^{-1}$ band activity is suppressed in $\mathrm{D}_{2} \mathrm{O}$ and its intensity is distributed over a number of other nearby modes. The isotope study (Figure 2) showed the $1220 \mathrm{~cm}^{-1}$ band to comprise at least a doublet, and three modes were calculated to be able to contribute. Although the $\mathrm{N} 3 \mathrm{H}$ wag is not contributing in these modes, for mode 75 it becomes prominent following exchange to N3D. This change is accompanied by a large blue shift (the resulting change in mode displacement is illustrated in supporting information). This is consistent with observation.

Turning to the triplet state (Figure 3c,d), the measurements show that the bands observed at 1519 and $1399 \mathrm{~cm}^{-1}$ are insensitive, while the lower wavenumbers bands $\left(1284\right.$ and $1202 \mathrm{~cm}^{-1}$ ) are sensitive, to N3H/D exchange. The calculation indicates that the carbonyl modes $\left(>1600 \mathrm{~cm}^{-1}\right)$ are strongly perturbed by both N3H/D exchange and H/D-bonding, as was the case for $\mathrm{S}_{0}$ and $\mathrm{S}_{1}$ (above). The three modes which can contribute to the observed $1519 \mathrm{~cm}^{-1}$ band are sensitive to N3H/D exchange, consistent with two of them (90 and 96 - see above) containing a significant displacement in $\mathrm{N} 3 \mathrm{H}$ wag. However, the observed effect is that these changes cancel one another out, leading to no overall shift. The $1284 \mathrm{~cm}^{-1}$ mode was unassigned on the basis of the calculations but is observed to be sensitive to exchange. The interesting case is the behaviour of the single mode 73 (Table 1 ) to which the $1202 \mathrm{~cm}^{-1}$ band could be assigned. In the measurements this mode is suppressed in $\mathrm{D}_{2} \mathrm{O}$. In the calculation its amplitude is also reduced upon N3H/D exchange, and another mode appears at lower wavenumber $\left(1168 \mathrm{~cm}^{-1}\right)$. When the $\mathrm{H}$-bonds are exchanged for deuterium bonds there is a small enhancement in the intensity of a previously very weak mode at $1228 \mathrm{~cm}^{-1}$, which may contribute to the observed red shift in the $1284 \mathrm{~cm}^{-1}$ mode.

In summary, the excited state modes of FMN are sensitive to exchange of $\mathrm{H}_{2} \mathrm{O}$ for $\mathrm{D}_{2} \mathrm{O}$. Marked effects were expected in the carbonyl stretch/N3H wag region, on the basis of earlier studies in $\mathrm{S}_{0}$. This is supported by calculation, but those effects will be most apparent in IR measurements. Concerning the FSRS data, the dominant deuteration effect is observed around $1200 \mathrm{~cm}^{-1}$ for both $\mathrm{S}_{1}$ and $T_{1}$ states. The underlying assignment involves a number of modes, which are mainly influenced 
by $\mathrm{N} 3 \mathrm{H} / \mathrm{D}$ exchange, rather than the $\mathrm{H}$-bonding environment. This suggests that the effect can be used as a marker for the rate of H/D exchange in Raman studies of flavoproteins.

Singlet - Triplet Spectral Shift. Here we consider changes in the spectra between the singlet and triplet states, which were already apparent in the FSRS data in Figure 2. Related spectral shifts have been reported in transient IR studies of FMN on singlet to triplet state conversion. ${ }^{9}$ We thus include the measured and calculated IR spectra in this section, and to allow this comparison we focus on calculations for N3D isoalloxazine in $\mathrm{D}_{2} \mathrm{O}$, the conditions used for IR measurements. We necessarily adopt a more qualitative approach to the comparison between theory and experiment, since for each new state a new set of modes is obtained. As a result, we cannot formally track individual modes between $S_{0}, S_{1}$ and $T_{1}$ states in the same way as was done for the different isotopologues (Figure 2). However, the geometries of $S_{1}$ and $T_{1}$ are very similar making some comparison of their modes meaningful.

Considering the FSRS data (Figure 4a,c) we note that the very broad shoulder to the blue of the 1508 $\mathrm{cm}^{-1}$ band in $\mathrm{S}_{1}$ has decreased in amplitude in $\mathrm{T}_{1}$, which is consistent with the calculated behaviour of as described above. The $1508 \mathrm{~cm}^{-1}$ band itself blue shifts on triplet state formation (as also reported by Fuertes and co-workers ${ }^{23}$ ) to $1518 \mathrm{~cm}^{-1}$. Comparing this to the calculations, we see that the blue shift is reproduced by an enhancement in the intensity of a mode at $1538 \mathrm{~cm}^{-1}$ localised on rings I and II, and a small blue shift in the intense $S_{1}$ mode localized on the same rings at $1509 \mathrm{~cm}^{-1}$ (see dashed lines in Figure 4c and for more detail SI). In the measured FSRS data the 1387 and $1430 \mathrm{~cm}^{-1}$ bands in $\mathrm{S}_{1}$ collapse to a single band in $\mathrm{T}_{1}$ at $1399 \mathrm{~cm}^{-1}$. This is not consistent with calculation, and indeed even its opposite. Both $S_{0}$ and $S_{1}$ have strong calculated Raman activity between 1300 and $1400 \mathrm{~cm}^{-1}$ and the $S_{0}$ data are consistent with both Raman and resonance Raman experiments. We 
(a) Experimental FSRS

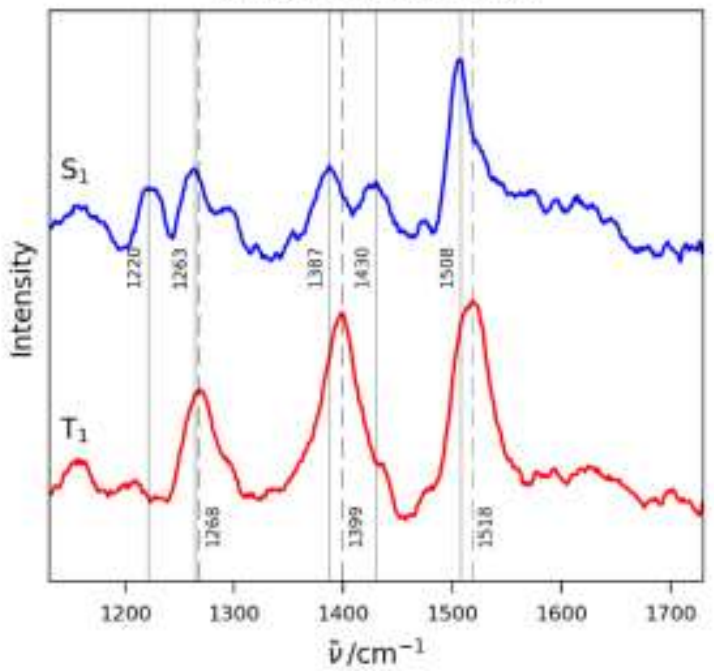

(c) Calculated RAMAN

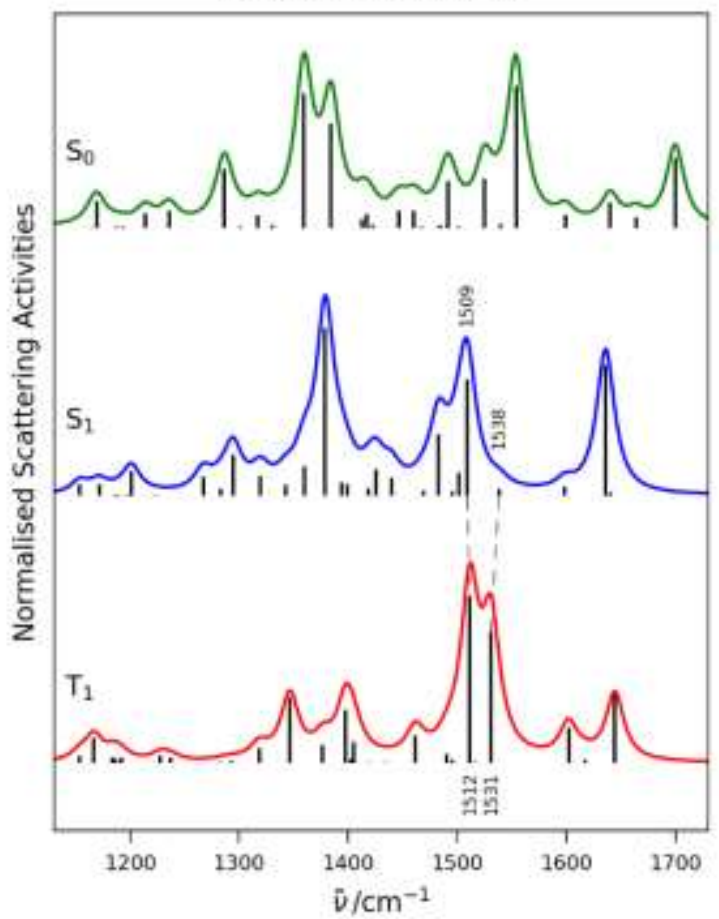

(b) Experimental TRIR

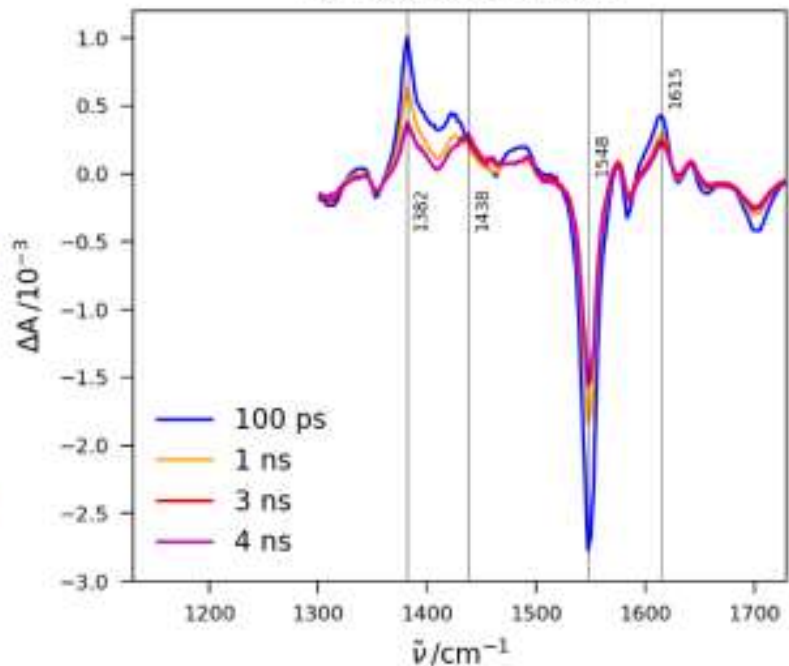

(d) Calculated IR

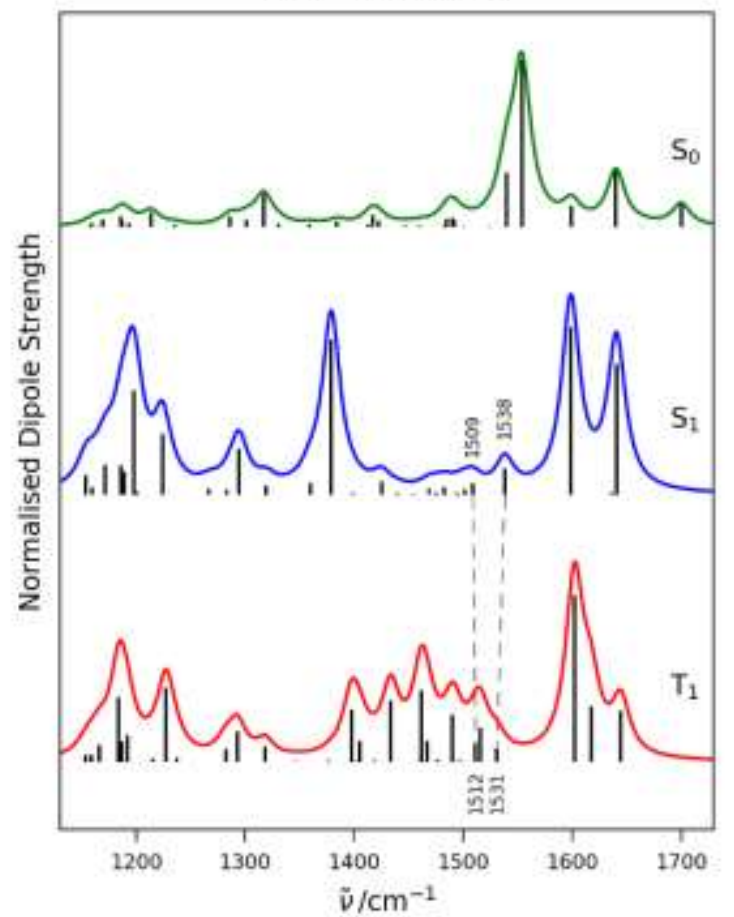

Figure 4: (a) $S_{1}$ (blue) and $T_{1}$ (red) FSRS spectra of FMN in $D_{2} O$. (b) TRIR spectra of FMN at 100 ps, 1 ns, 3 ns and $4 \mathrm{~ns}$ (deuterated buffer, $20 \mathrm{mM}$ Tris hydrochloride, $\mathrm{pH}$ 8.0, containing $150 \mathrm{mM} \mathrm{NaCl}$ ). (c) Calculated Raman and (d) IR spectra of FMN with N3D + D2O substitution for $S_{0}$ (green), $S_{1}$ (blue) and $T_{1}$ (red).

For the IR data (Figure 4b,d) there are also points of agreement and disagreement with calculation.

First the TRIR data in Figure 4b show the time resolved IR spectra of FMN evolving from initial population of the $S_{1}$ state following ground state excitation $(t=0)$, which then evolves in 
nanoseconds to form the $T_{1}$ state. Since these are difference spectra, the $S_{0}$ data are also included as bleaches (negative optical density, OD) while the transient (positive OD) represents formation of the $\mathrm{S}_{1}$ and $\mathrm{T}_{1}$ states in the picosecond and nanosecond spectra respectively. The bleach features match the calculated $S_{0}$ IR spectra well, as previously noted. ${ }^{15-16,54}$ The two highest wavenumber modes arise from separate $\mathrm{C}=\mathrm{O}+\mathrm{N} 3 \mathrm{D}$ wag, and the next two lowest wavenumber modes are ring modes involving $\mathrm{C}=\mathrm{N}$ stretches. Upon electronic excitation the bleaches at $1650-1700 \mathrm{~cm}^{-1}\left(\mathrm{~S}_{0}\right)$ are accompanied by formation of a weak $\mathrm{S}_{1}$ positive feature at $1615 \mathrm{~cm}^{-1}$. In terms of wavenumber this corresponds with the calculated carbonyl modes in the excited state at 1599 and $1636 \mathrm{~cm}^{-1}$, but the intensities differ, the measured signal being much weaker than that calculated. The intense bleach of the ring mode at $1548 \mathrm{~cm}^{-1}$ is not accompanied by a strong positive feature in the $\mathrm{S}_{1}$ spectrum. This is in good agreement with calculation, where there is no corresponding intense feature in the calculated $S_{1}$ spectrum. As the $S_{1}$ state decays to $T_{1}$ the most remarkable change in the IR spectra is the shift from $1382 \mathrm{~cm}^{-1}$ absorption to $1438 \mathrm{~cm}^{-1}$. Again this accords nicely with calculation, where a strong IR mode at $1379 \mathrm{~cm}^{-1}$ in $S_{1}$ is replaced by a complex set of modes between 1400 and 1500 $\mathrm{cm}^{-1}$ in $\mathrm{T}_{1}$.

Aqueous Solution - Protein Spectral Shifts. In this section we compare the $S_{1}$ and $T_{1}$ Raman spectra of FMN in buffer solution with those measured for FMN in AsLOV2. Time resolved IR studies of AsLOV2 have been reported previously, ${ }^{25,55}$ but this is the first time-resolved Raman study. It is particularly important to characterise the FMN triplet state of LOV domains, since this is the reactive precursor leading to formation of the adduct state on the microsecond timescale, ${ }^{24}$ which triggers the structure change which in turn results in the signalling state. When this reaction, which occurs between the triplet FMN and an adjacent cysteine residue, is blocked the FMN triplet state is formed in high yield and has been shown to act as a genetically expressible source of reactive oxygen. ${ }^{56-57}$ Figure 5 shows FSRS data for FMN and AsLOV2 recorded as a function of time, revealing the expected evolution from excited singlet to triplet state on the nanosecond time scale. Qualitatively, there is a high degree of similarity between spectra measured in the two environments. This 

contrasts with time resolved IR studies of AsLOV2 and FMN, where additional features in AsLOV2 are observed on all time scales and have been assigned to excitation induced changes in the IR spectra of interacting amino acid residues. ${ }^{9,14}$ This difference is assumed to arise because the FSRS signals are enhanced by resonance with electronic transitions, which are localised on the chromophore. 
(a) FSRS

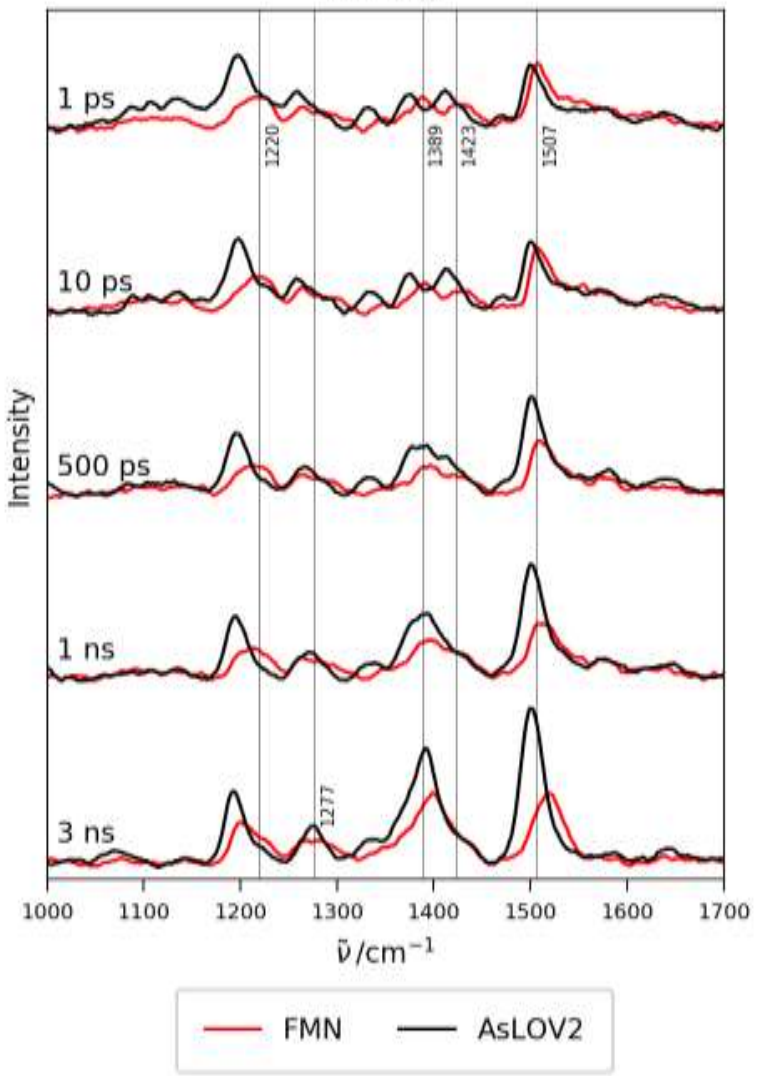

(b) ligplot

Figure 5: (a) FSRS spectra of aqueous FMN (red) and AsLOV2 (black) in $20 \mathrm{mM}$ Tris hydrochloride, $\mathrm{pH}$ 8.0, containing $150 \mathrm{mM} \mathrm{NaCl}$ at $1 \mathrm{ps}\left(\mathrm{S}_{1}\right), 10 \mathrm{ps}, 500 \mathrm{ps}, 1 \mathrm{~ns}$ and $3 \mathrm{~ns}\left(\mathrm{~T}_{1}\right)$. (b) ligplot analysis of AsLOV2 interactions in the FMN binding pocket.

261 There are however significant differences in the details of FMN and AsLOV2 FSRS spectra (both measured in $\mathrm{H}_{2} \mathrm{O}$ buffer). In the $1200 \mathrm{~cm}^{-1}$ region, the $1220 \mathrm{~cm}^{-1}$ band in solution is red shifted in the 
protein, and better resolved. In both environments the band shifts to the red on formation of the triplet, but the shift is smaller in the protein. In both samples a new band appears in the triplet state spectra, most clearly resolved at $1277 \mathrm{~cm}^{-1}$ for AsLOV2. The band structure in the $1200 \mathrm{~cm}^{-1}$ region was shown to be sensitive to $\mathrm{N} 3 \mathrm{H} / \mathrm{D}$ exchange and $\mathrm{H}$-bonding environment, so we speculate that differing interactions between $\mathrm{FMN}$ at $\mathrm{N} 3 \mathrm{H}$ and either $\mathrm{H}_{2} \mathrm{O}$ or the amino acid residues in the binding site are the origin of the behaviour observed.

For both samples there is a complex spectrum in the $S_{1}$ state between 1330 and $1450 \mathrm{~cm}^{-1}$, involving three bands. These bands in AsLOV2 are red shifted compared to FMN in solution. For both samples this band structure evolves into a single strong, broad and asymmetric band in the $T_{1}$ state, again slightly red shifted in the protein. The most surprising result in Figure 5 is the absence in AsLOV2 of Finally, we assess the use of isotope labelling in assigning vibrational bands in protein excited state Raman spectra. To this ends we compare FSRS of AsLOV2 with AsLOV2 loaded with [U- $\left.{ }^{15} \mathrm{~N}_{4}\right]-\mathrm{FMN}$ example, the $7 \mathrm{~cm}^{-1}$ shift in the $1501 \mathrm{~cm}^{-1}$ band and the $17 \mathrm{~cm}^{-1}$ red shift in the $1391 \mathrm{~cm}^{-1}$ band agree well with solution data for $\left[\mathrm{U}_{-15}^{15} \mathrm{~N}_{4}\right]-\mathrm{FMN}$ and with calculations (Figure 2). For the $\mathrm{S}_{1}$ state the small (2 $\mathrm{cm}^{-1}$ ) of shift in the $1499 \mathrm{~cm}^{-1}$ band also correlates with calculated FMN data, as does the red shift of the $1412 \mathrm{~cm}^{-1}$ band. However, the downshift of the $1197 \mathrm{~cm}^{-1}$ band in $\left[\mathrm{U}^{-15} \mathrm{~N}_{4}\right]-\mathrm{FMN}$ is larger than in 

surrounding residues.

(a) $\mathrm{S}_{1}$



(b) $T_{1}$

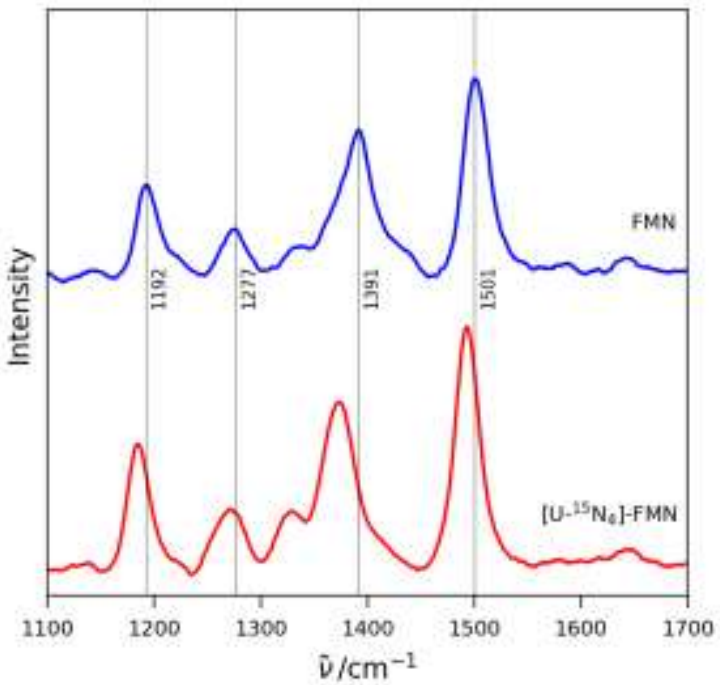

292

293

294

295

296

297

298

299

300

301

302

Figure 6: FSRS spectra of aqueous (20 mM Tris hydrochloride, $\mathrm{pH}$ 8.0, containing $150 \mathrm{mM} \mathrm{NaCl)} \mathrm{AsLOV2}$ containing FMN (blue) and [U- $\left.{ }^{15} N_{4}\right]-F M N\left(\right.$ red) (a) $S_{1}$; (b) $T_{1}$.

Conclusions. The excited state Raman spectra of the singlet and triplet states of FMN have been measured by resonant FSRS in solution and in AsLOV2. The measurements have been extended to several FMN isotopologues, and the data are compared with DFT and TD-DFT calculations of excited state vibrational spectra. The measured spectra are in general simpler than the calculated spectra, probably because FSRS is a resonant experiment, and only a subset of Raman active modes gain from resonance enhancement; in particular, the carbonyl localised modes are very weak in the FSRS data.

The observed isotope shifts for the $S_{1}$ and $T_{1}$ states of $F M N$ in aqueous solution are generally well reproduced by the calculations, although multiple modes contribute to the observations, which complicates assignment. In general, the resonant FSRS data are dominated by ring modes. However, experiment and calculation for the effects of deuteration showed that exchange at N3H/D has a significant effect on a number of Raman active modes, an effect that could be used to investigate 
isotope exchange rates in flavoproteins. FSRS measurements were extended to the LOV domain protein AsLOV2 and it was shown that the FSRS spectra are dominated by chromophore localised modes (a consequence of resonance enhancement) and that differential interactions with the environment led to some changes in the observed spectra.

Raman spectroscopy has many advantages over IR as a tool for the study of biomolecules - it is not restricted to $D_{2} \mathrm{O}$ solutions and can be observed even for large proteins and their complexes. Further, a broad wavenumber range is observed in the Raman measurement, in contrast to transient IR experiments which may be limited by the IR bandwidth available. In ultrafast photobiology, the Raman spectrum of a specific (resonant) transient excited state can be measured, a degree of selectivity not available in transient IR. The present work shows that measurement and assignment of excited state Raman spectra can be undertaken in flavoproteins, thus opening the way to the more widespread application of FSRS to probe structural dynamics in photobiology.

\section{Acknowledgements}

This study was supported by the National Science Foundation (NSF) (MCB-1817837 to PJT, MCB1750637 to J.B.F.) and the EPSRC (EP/N033647/1 to SRM). JNI was supported by a National Institutes of Health Chemistry-Biology Interface Training Grant (T32GM092714). AL acknowledges funding from EFOP-3.6.2-16-2017-00005. JBF acknowledges the Research Corporation for Science Advancement for a Cottrell Scholar Award. The calculations presented in this paper was carried out on the High Performance Computing Cluster supported by the Research and Specialist Computing Support service at the University of East Anglia. 
332 1. Massey, V., The Chemical and Biological Versatility of Riboflavin. Biochem. Soc. Trans. 2000, $33328,283-296$.

$3342 . \quad$ Losi, A.; Gartner, W., The Evolution of Flavin-Binding Photoreceptors: An Ancient Chromophore Serving Trendy Blue-Light Sensors. In Annual Review of Plant Biology, Vol 63, Merchant, S. S., Ed. 2012; Vol. 63, pp 49-72.

3. Sancar, A., Structure and Function of DNA Photolyase and Cryptochrome Blue-Light Photoreceptors. Chemical Reviews 2003, 103, 2203-2237.

4. Fiedler, B.; Borner, T.; Wilde, A., Phototaxis in the Cyanobacterium Synechocystis Sp Pcc 6803: Role of Different Photoreceptors. Photochem. Photobiol. 2005, 81, 1481-1488.

5. Christie, J. M., Phototropin Blue-Light Receptors. Annual Review of Plant Biology 2007, 58, 21-45.

6. Losi, A.; Gardner, K. H.; Moglich, A., Blue-Light Receptors for Optogenetics. Chemical Reviews 2018, 118, 10659-10709.

7. Brazard, J.; Usman, A.; Lacombat, F.; Ley, C.; Martin, M. M.; Plaza, P., New Insights into the Ultrafast Photophysics of Oxidized and Reduced Fad Ins Solution. J. Phys. Chem. A, 115, 3251-3262. 8. Kennis, J. T. M.; Groot, M. L., Ultrafast Spectroscopy of Biological Photoreceptors. Current Opinion in Structural Biology 2007, 17, 623-630.

9. Brust, R.; Lukacs, A.; Haigney, A.; Addison, K.; Gil, A.; Towrie, M.; Clark, I. P.; Greetham, G. M.; Tonge, P. J.; Meech, S. R., Proteins in Action: Femtosecond to Millisecond Structural Dynamics of a Photoactive Flavoprotein. J. Am. Chem. Soc. 2013, 135, 16168-16174.

10. Stelling, A. L.; Ronayne, K. L.; Nappa, J.; Tonge, P. J.; Meech, S. R., Ultrafast Structural Dynamics in Bluf Domains: Transient Infrared Spectroscopy of Appa and Its Mutants. J. Am. Chem. Soc. 2007, 129, 15556-15564.

11. Kottke, T.; Heberle, J.; Hehn, D.; Dick, B.; Hegemann, P., Phot-Lov1: Photocycle of a BlueLight Receptor Domain from the Green Alga Chlamydomonas Reinhardtii. Biophys. J. 2003, 84, 11921201.

12. Mathes, T.; Zhu, J. Y.; van Stokkum, I. H. M.; Groot, M. L.; Hegemann, P.; Kennis, J. T. M., Hydrogen Bond Switching among Flavin and Amino Acids Determines the Nature of Proton-Coupled Electron Transfer in Bluf Photoreceptors. Journal of Physical Chemistry Letters 2012, 3, 203-208.

13. Mathes, T.; van Stokkum, I. H. M.; Stierl, M.; Kennis, J. T. M., Redox Modulation of Flavin and Tyrosine Determines Photoinduced Proton-Coupled Electron Transfer and Photoactivation of Bluf Photoreceptors. J. Biol. Chem. 2012, 287, 31725-31738.

14. Lukacs, A.; Haigney, A.; Brust, R.; Zhao, R. K.; Stelling, A. L.; Clark, I. P.; Towrie, M.; Greetham, G. M.; Meech, S. R.; Tonge, P. J., Photoexcitation of the Blue Light Using Fad Photoreceptor Appa Results in Ultrafast Changes to the Protein Matrix. J. Am. Chem. Soc. 2011, 133, 16893-16900. 15. Haigney, A., et al., Ultrafast Infrared Spectroscopy of an Isotope-Labeled Photoactivatable Flavoprotein. Biochemistry 2011, 50, 1321-1328.

16. Haigney, A., et al., Vibrational Assignment of the Ultrafast Infrared Spectrum of the Photoactivatable Flavoprotein Appa. J. Phys. Chem. B 2012, 116, 10722-10729.

17. Hall, C. R.; Collado, J. T.; Iuliano, J. N.; Gil, A. A.; Adamczyk, K.; Lukacs, A.; Greetham, G. M.; Sazanovich, I.; Tonge, P. J.; Meech, S. R., Site-Specific Protein Dynamics Probed by Ultrafast Infrared Spectroscopy of a Noncanonical Amino Acid. Journal of Physical Chemistry B 2019, 123, 9592-9597. 18. Kukura, P.; McCamant, D. W.; Mathies, R. A., Femtosecond Stimulated Raman Spectroscopy. Annual Review of Physical Chemistry 2007, 58, 461-488.

19. Frontiera, R. R.; Mathies, R. A., Femtosecond Stimulated Raman Spectroscopy. Laser \& Photonics Reviews 2011, 5, 102-113.

20. Zhu, L. D.; Liu, W. M.; Fang, C., A Versatile Femtosecond Stimulated Raman Spectroscopy Setup with Tunable Pulses in the Visible to near Infrared. Applied Physics Letters 2014, 105. 
21. Weigel, A.; Dobryakov, A.; Klaumunzer, B.; Sajadi, M.; Saalfrank, P.; Ernsting, N. P., Femtosecond Stimulated Raman Spectroscopy of Flavin after Optical Excitation. J. Phys. Chem. $B$ 2011, 115, 3656-3680.

22. Hall, C. R.; Heisler, I. A.; Jones, G. A.; Frost, J. E.; Gil, A. A.; Tonge, P. J.; Meech, S. R., Femtosecond Stimulated Raman Study of the Photoactive Flavoprotein Appa(Bluf). Chem. Phys. Lett. 2017, 683, 365-369.

23. Andrikopoulos, P. C., et al., Femtosecond-to-Nanosecond Dynamics of Flavin Mononucleotide Monitored by Stimulated Raman Spectroscopy and Simulations. Physical Chemistry Chemical Physics 2020, 22, 6538-6552.

24. Gil, A. A., et al., Femtosecond to Millisecond Dynamics of Light Induced Allostery in the Avena Sativa Lov Domain. J. Phys. Chem. B 2017, 121, 1010-1019.

25. Konold, P. E.; Mathes, T.; Weißenborn, J.; Groot, M. L.; Hegemann, P.; Kennis, J. T. M., Unfolding of the C-Terminal J $\alpha$ Helix in the Lov2 Photoreceptor Domain Observed by Time-Resolved Vibrational Spectroscopy. The Journal of Physical Chemistry Letters 2016, 3472-3476.

26. Schleicher, E.; Kowalczyk, R. M.; Kay, C. W. M.; Hegemann, P.; Bacher, A.; Fischer, M.; Bittl, R.; Richter, G.; Weber, S., On the Reaction Mechanism of Adduct Formation in Lov Domains of the Plant Blue-Light Receptor Phototropin. J. Am. Chem. Soc. 2004, 126, 11067-11076.

27. Harper, S. M.; Christie, J. M.; Gardner, K. H., Disruption of the Lov-J Alpha Helix Interaction Activates Phototropin Kinase Activity. Biochemistry 2004, 43, 16184-16192.

28. Moglich, A.; Moffat, K., Engineered Photoreceptors as Novel Optogenetic Tools. Photochemical \& Photobiological Sciences 2010, 9, 1286-1300.

29. Dagliyan, O.; Tarnawski, M.; Chu, P.-H.; Shirvanyants, D.; Schlichting, I.; Dokholyan, N. V.; Hahn, K. M., Engineering Extrinsic Disorder to Control Protein Activity in Living Cells. Science 2016, $354,1441$.

30. Hoffmann, M. D.; Mathony, J.; Upmeier zu Belzen, J.; Harteveld, Z.; Stengl, C.; Correia, B. E.; Eils, R.; Niopek, D., Optogenetic Control of \&Lt;Em\&Gt;Neisseria Meningitidis\&Lt;/Em\&Gt; Cas9 Genome Editing Using an Engineered, Light-Switchable Anti-Crispr Protein. bioRxiv 2019, 858589. 31. Hall, C. R.; Conyard, J.; Heisler, I. A.; Jones, G.; Frost, J.; Browne, W. R.; Feringa, B. L.; Meech, S. R., Ultrafast Dynamics in Light-Driven Molecular Rotary Motors Probed by Femtosecond Stimulated Raman Spectroscopy. J. Am. Chem. Soc. 2017, 139, 7408-7414.

32. Heisler, I. A.; Moca, R.; Camargo, F. V. A.; Meech, S. R., Two-Dimensional Electronic Spectroscopy Based on Conventional Optics and Fast Dual Chopper Data Acquisition. Rev. Sci. Instrum. 2014, 85, 10.

33. Becke, A. D., Density-Functional Thermochemistry. lii. The Role of Exact Exchange. The Journal of Chemical Physics 1993, 98, 5648-5652.

34. Lee, C.; Yang, W.; Parr, R. G., Development of the Colle-Salvetti Correlation-Energy Formula into a Functional of the Electron Density. Physical Review B 1988, 37, 785-789.

35. Wetmore, S. D.; Huang, Y., Looking Back on 90 Years of the Canadian Journal of Chemistry. Canadian Journal of Chemistry 2019, 97, iii-iv.

36. Scalmani, G.; Frisch, M. J.; Mennucci, B.; Tomasi, J.; Cammi, R.; Barone, V., Geometries and Properties of Excited States in the Gas Phase and in Solution: Theory and Application of a TimeDependent Density Functional Theory Polarizable Continuum Model. The Journal of Chemical Physics 2006, 124, 094107.

37. Tomasi, J.; Mennucci, B.; Cammi, R., Quantum Mechanical Continuum Solvation Models. Chemical Reviews 2005, 105, 2999-3094.

38. Zenichowski, K.; Gothe, M.; Saalfrank, P., Exciting Flavins: Absorption Spectra and Spin-Orbit Coupling in Light-Oxygen-Voltage (Lov) Domains. Journal of Photochemistry and Photobiology A: Chemistry 2007, 190, 290-300.

39. Brealey, G. J.; Kasha, M., The Role of Hydrogen Bonding in the N-Pi-Star Blue-Shift Phenomenon. Journal of the American Chemical Society 1955, 77, 4462-4468.

40. Frisch, M. J., et al., Gaussian 09, Revision B.01. Wallingford CT, 2009. 
41. Tishler, M.; Pfister, K.; Babson, R. D.; Ladenburg, K.; Fleming, A. J., The Reaction between OAminoazo Compounds and Barbituric Acid. A New Synthesis of Riboflavin. Journal of the American Chemical Society 1947, 69, 1487-1492.

42. Illarionov, B.; Fischer, M.; Lee, C. Y.; Bacher, A.; Eisenreich, W., Rapid Preparation of Isotopolog Libraries by in Vivo Transformation of C-13-Glucose. Studies on 6,7-Dimethyl-8Ribityllumazine, a Biosynthetic Precursor of Vitamin B-2. J. Org. Chem. 2004, 69, 5588-5594. 43. Pompe, N.; Chen, J.; Illarionov, B.; Panter, S.; Fischer, M.; Bacher, A.; Weber, S., Methyl Groups Matter: Photo-Cidnp Characterizations of the Semiquinone Radicals of Fmn and Demethylated Fmn Analogs. The Journal of Chemical Physics 2019, 151, 235103.

44. Hall, C. R.; Romanov, A. S.; Bochmann, M.; Meech, S. R., Ultrafast Structure and Dynamics in the Thermally Activated Delayed Fluorescence of a Carbene-Metal-Amide. Journal of Physical Chemistry Letters 2018, 9, 5873-5876.

45. Holzer, W.; Penzkofer, A.; Fuhrmann, M.; Hegemann, P., Spectroscopic Characterization of Flavin Mononucleotide Bound to the Lov1 Domain of Phot1 from Chlamydomonas Reinhardtii Photochemistry and Photobiology 2002, 75, 479-487.

46. Islam, S. D. M.; Penzkofer, A.; Hegemann, P., Quantum Yield of Triplet Formation of Riboflavin in Aqueous Solution and of Flavin Mononucleotide Bound to the Lov1 Domain of Phot1 from Chlamydomonas Reinhardtii. Chemical Physics 2003, 291, 97-114.

47. Losi, A.; Polverini, E.; Quest, B.; Gärtner, W., First Evidence for Phototropin-Related BlueLight Receptors in Prokaryotes. Biophysical Journal 2002, 82, 2627-2634.

48. Quincy, T. J.; Barclay, M. S.; Caricato, M.; Elles, C. G., Probing Dynamics in Higher-Lying Electronic States with Resonance-Enhanced Femtosecond Stimulated Raman Spectroscopy. The Journal of Physical Chemistry A 2018, 122, 8308-8319.

49. Kitagawa, T.; Nishina, Y.; Kyogoku, Y.; Yamano, T.; Ohishi, N.; Takaisuzuki, A.; Yagi, K., Resonance Raman-Spectra of Carbon-13-Labeled and Nitrogen-15-Labeled Riboflavin Bound to EggWhite Flavoprotein. Biochemistry 1979, 18, 1804-1808.

50. Iwata, T.; Nozaki, D.; Sato, Y.; Sato, K.; Nishina, Y.; Shiga, K.; Tokutomi, S.; Kandori, H., Identification of the Co Stretching Vibrations of Fmn and Peptide Backbone by 13c-Labeling of the Lov2 Domain of Adiantum Phytochrome3. Biochemistry 2006, 45, 15384-15391.

51. Halavaty, A. S.; Moffat, K., N- and C-Terminal Flanking Regions Modulate Light-Induced Signal Transduction in the Lov2 Domain of the Blue Light Sensor Phototropin 1 from Avena Sativa. Biochemistry 2007, 46, 14001-14009.

52. Unno, M.; Masuda, S.; Ono, T. A.; Yamauchi, S., Orientation of a Key Glutamine Residue in the Bluf Domain from Appa Revealed by Mutagenesis, Spectroscopy, and Quantum Chemical Calculations. J. Am. Chem. Soc. 2006, 128, 5638-5639.

53. Hazekawa, I.; Nishina, Y.; Sato, K.; Shichiri, M.; Miura, R.; Shiga, K., A Raman Study on the $C(4)=O$ Stretching Mode of Flavins in Flavoenzymes: Hydrogen Bonding at the $C(4)=0$ Moiety. Journal of Biochemistry 1997, 121, 1147-1154.

54. Kondo, M.; Nappa, J.; Ronayne, K. L.; Stelling, A. L.; Tonge, P. J.; Meech, S. R., Ultrafast Vibrational Spectroscopy of the Flavin Chromophore. J. Phys. Chem. B 2006, 110, 20107-20110. 55. Iuliano, J. N., et al., Variation in Lov Photoreceptor Activation Dynamics Probed by TimeResolved Infrared Spectroscopy. Biochemistry 2017.

56. Pimenta, F. M.; Jensen, R. L.; Breitenbach, T.; Etzerodt, M.; Ogilby, P. R., Oxygen-Dependent Photochemistry and Photophysics of "Minisog," a Protein-Encased Flavin. Photochemistry and Photobiology 2013, 89, 1116-1126.

57. Shu, X. K.; Lev-Ram, V.; Deerinck, T. J.; Qi, Y. C.; Ramko, E. B.; Davidson, M. W.; Jin, Y. S.; Ellisman, M. H.; Tsien, R. Y., A Genetically Encoded Tag for Correlated Light and Electron Microscopy of Intact Cells, Tissues, and Organisms. Plos Biology 2011, 9.

58. Wallace, A. C.; Laskowski, R. A.; Thornton, J. M., Ligplot: A Program to Generate Schematic Diagrams of Protein-Ligand Interactions. Protein engineering 1995, 8, 127-34. 


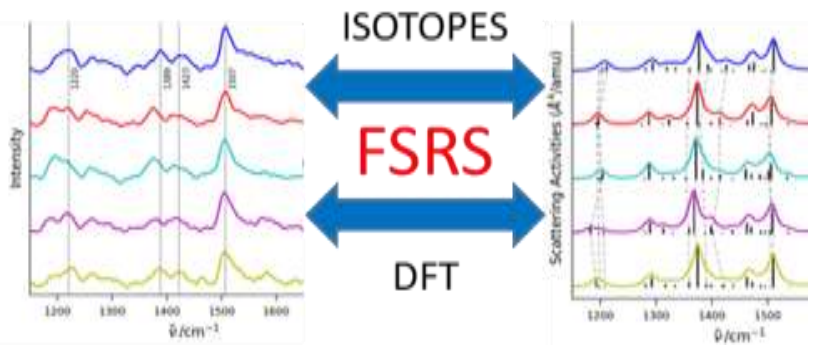

